\title{
Is there any place for PD-1/CTLA-4 inhibitors combination in the first-line treatment of advanced NSCLC? - a trial-level meta-analysis in PD-L1 selected subgroups
}

\author{
Francesco Passiglia ${ }^{1 \#}$, Antonio Galvano ${ }^{2 \#}$, Valerio Gristina ${ }^{2 \#}$, Nadia Barraco ${ }^{2}$, Marta Castiglia $^{2}$, \\ Alessandro Perez ${ }^{2}$, Maria La Mantia ${ }^{2}$, Antonio Russo ${ }^{2 *}$, Viviana Bazan ${ }^{3 *}$ \\ ${ }^{1}$ Department of Oncology, University of Turin, San Luigi Gonzaga Hospital, Regione Gonzole 10, 10043, Orbassano, Turin, Italy; ${ }^{2}$ Department \\ of Surgical, Oncological and Oral Sciences, University of Palermo, Palermo, Italy; ${ }^{3}$ Department of Experimental Biomedicine and Clinical \\ Neurosciences, University of Palermo, Palermo, Italy \\ Contributions: (I) Conception and design: F Passiglia, A Galvano, V Gristina; (II) Administrative support: V Bazan, A Russo; (III) Provision of study \\ materials or patients: N Barraco, A Perez, M Castiglia, M La Mantia; (IV) Collection and assembly of data: F Passiglia, A Galvano, V Gristina; \\ (V) Data analysis and interpretation: F Passiglia, A Galvano, V Gristina, A Russo; (VI) Manuscript writing: All authors; (VII) Final approval of \\ manuscript: All authors. \\ \#These authors contributed equally to this work. \\ *These authors are equal co-last authors. \\ Correspondence to: Prof. Antonio Russo. Section of Medical Oncology, Department of Surgical, Oncological and Oral Sciences, Via del Vespro 129, \\ 90127, University of Palermo, Palermo, Italy. Email: antonio.russo@unipa.it.
}

Background: The advent of immuno-oncology (IO) represented a breakthrough in non-small cell lung cancer (NSCLC) therapy over the last few years. However, establishing the optimal therapeutic options among programmed death-ligand 1 (PD-L1) selected subgroups still addresses an unmet need in the clinical setting.

Methods: We performed a systematic review and finally included eleven first-line randomized controlled trials to compare efficacy and safety outcomes among first-line IO treatment strategies versus standard platinum-based chemotherapy (CT) according to PD-L1 expression level $(<1 \%, 1-49 \%, \geq 50 \%)$. Pooled hazard ratios (HRs) and risk ratios (RRs) for progression-free survival (PFS), overall survival (OS), objective response rates (ORR), treatment-related adverse events (TRAEs), and discontinuation rates were obtained.

Results: Our results demonstrated that among the different IO-based strategies (single-agent IO, Combo$\mathrm{IO}, \mathrm{IO}+\mathrm{CT})$ the $\mathrm{IO}+\mathrm{CT}$ approach resulted in a significant increase of the ORR, albeit with no relevant improvement of survival in patients with PD-L1 $\geq 50 \%$. As regards patients with negative PD-L1 expression, no significant differences in terms of activity and efficacy profile have been detected between the IO + CT and the dual checkpoint blockade. Of note, in the PD-L1 1-49\% subgroup, the use of anti-PD-1 agents in association with CT led to a statistically significant gain in OS. As concerns safety, the dual checkpoint blockade seemed to be better tolerated than $\mathrm{IO}+\mathrm{CT}$.

Conclusions: This meta-analysis suggested the current limited role of PD-1/CTLA-4 inhibitors combination in PD-L1-high and/or -low advanced NSCLC patients while emerging as a potentially effective and tolerable option in particular PD-L1 negative subgroups.

Keywords: Non-small cell lung cancer (NSCLC); programmed death-1/cytotoxic T-lymphocyte antigen 4 inhibitors (PD-1/CTLA-4 inhibitors); immunotherapy; combined modality therapy; meta-analysis

Submitted Jan 22, 2021. Accepted for publication Apr 06, 2021.

doi: $10.21037 /$ tlcr-21-52

View this article at: https://dx.doi.org/10.21037/tlcr-21-52 


\section{Introduction}

The advent of immune-oncology (IO), particularly the immune-checkpoint inhibitors (ICIs) targeting the programmed death-1 (PD-1)/programmed death ligand-1 (PD-L1) axis, represented a breakthrough in lung cancer therapy over the last few years. The introduction of IO monotherapy has initially revolutionized the second-line treatment of advanced non-small cell lung cancer (NSCLC), leading to a significant increase of 5-year survival, reaching $16 \%$ nowadays, compared to $5.5 \%$ in the chemotherapy (CT) era (1). The anti-PD-1 agent pembrolizumab demonstrated to significantly improve progression-free survival (PFS) as well as overall survival (OS) and quality of life as compared to platinum-CT in the first-line treatment of non-oncogene addicted NSCLC with tumor PD-L1 expression higher than $50 \%$, representing the current upfront standard for about $30 \%$ of patients with newly diagnosed metastatic disease (2). More recently, upfront combination studies, evaluating the addition of IO to platinum-based CT-regimens, have proven to synergistically enhance individual immune response, eventually leading to improved clinical outcomes, thus obtaining regulatory approval regardless of PD-L1 expression levels $(3,4)$. Different meta-analyses have indirectly compared the $\mathrm{IO}+$ CT combination strategies versus IO alone in the subgroup of patients with high PD-L1 expression, revealing only a significant increase of activity [higher objective response rate (ORR) and prolonged PFS], without any OS benefit in favor of the combination regimens (5).

Recently, the combination of different classes of ICIs harboring complementary mechanisms of action, such as the anti-PD-1/PD-L1 agents with the cytotoxic T-lymphocyte antigen 4 (CTLA-4) inhibitors, has significantly aroused interest in terms of improved efficacy in selected populations, albeit with an expected increased rate of adverse events. Specifically, the combination of nivolumab and ipilimumab showed to significantly improve OS as compared to platinum-CT in PD-L1 $\geq 1 \%$ advanced NSCLC, with exploratory analysis showing a survival benefit also in PD-L1-negative patients, thus emerging as an additional upfront therapeutic option, at least in United States (6).

To date, we have three different immune-based treatment regimens, including single-agent $\mathrm{IO}, \mathrm{IO}+\mathrm{CT}$, and $\mathrm{IO}+$ $\mathrm{IO}$, all showing a significant superiority in terms of activity and long-term survival over platinum-CT for the upfront treatment of non-oncogene addicted metastatic NSCLC patients (7). Namely, all these three strategies are potentially available for the treatment of the PD-L1 high subgroup of patients. On the other hand, despite the currently limited survival data only IO-CT and PD-1-CTLA-4 inhibitors combinations have been approved for the subgroup of patients with low or negative PD-L1 expression, even if in this setting the Food and Drug Administration has recently granted an expanded monotherapy indication for pembrolizumab. Considering the lack of head-tohead comparisons between the aforementioned treatment approaches, establishing the optimal therapeutic options among advanced NSCLC patients' subgroups still addresses an unmet need in the clinical setting. So far, the immunohistochemical evaluation of PD-L1 expression on tumor cells has been the most studied IO predictive biomarker, since a coherent correlation between PD-L1 expression and treatment efficacy has emerged among all these clinical trials.

Here we conducted an indirect meta-analysis of randomized trials to comprehensively compare activity, efficacy along the major safety outcomes among these three different immune-based treatment strategies within the main PD-L1 selected subgroups (PD-L1 negative, low or high) of naive patients with advanced NSCLC. The current meta-analysis aims to strengthen clinically useful evidence and to provide a deeper insight into potential efficacy and tolerability differences regarding the three different firstline immune-based treatment options within the three major PD-L1 expression subgroups of metastatic NSCLC. We present the following article in accordance with the PRISMA reporting checklist (available at https://dx.doi. org/10.21037/tlcr-21-52).

\section{Methods}

\section{Search strategy and study}

We searched for first-line randomized controlled trials (RCTs) comparing single-agent IO (IO) versus platinum-doublet combination regimens $(\mathrm{CT})$ or $\mathrm{IO}+\mathrm{CT}$ versus $\mathrm{CT}$ or IO + IO (Combo IO) versus CT in patients with histologically proven diagnosis of unresectable or advanced (Stage IIIB/ C-IV) non-oncogene addicted NSCLC. We included RCTs comparing the association of combination ICIs with CT vs. CT alone in the $\mathrm{IO}+\mathrm{CT}$ subgroup. RCTs focusing on the adjuvant/neoadjuvant setting (Stage I-IIIA NSCLC) were excluded. Moreover, we excluded non-randomized or cross-sectional, case-control, cohort, or retrospective 
studies. We also excluded studies with unavailable outcomes, ongoing clinical trials as well as studies with small sample size (less than 10 patients for arm). Namely, sixteen cohorts from fourteen different randomized controlled studies [KEYNOTE-024 (8), KEYNOTE-042 (2), IMpower110 (9), CheckMate-227 part 1-2 (10,11), EMPOWER-Lung 1 (12), KEYNOTE-189 (13), KEYNOTE-407 (3), IMpower150 (4), IMpower130 (14), IMpower131 (15), IMpower132 (16), CheckMate-9LA (17), MYSTIC (18)] have been included in the final analysis.

As IO we included pembrolizumab, cemiplimab or nivolumab (PD-1 group) or atezolizumab or durvalumab (PD-L1 group). As Combo IO we included nivolumab + ipilimumab or durvalumab + tremelimumab combinations. As $\mathrm{IO}+$ CT we included atezolizumab +/- bevacizumab, pembrolizumab or nivolumab with or without ipilimumab in combination with standard CT. As standard CT we included cisplatin or carboplatin in association with gemcitabine or paclitaxel or pemetrexed, or nab-paclitaxel, according to NSCLC histology (squamous or nonsquamous cell carcinoma).

We used specific keywords and Boolean operators (Figure S1). Data available up to 10 March 2021 on Medline (PubMed), Scopus, and Cochrane-Library database were collected, without language restrictions; relevant abstracts published on the American Society of Clinical Oncology (ASCO) and the European Society of Medical Oncology (ESMO) databases, as well as unpublished data or results from ongoing studies available on the National Institute of Health (NIH) website (www.clinicaltrials.gov) were also considered as a source of grey literature. We submitted our systematic review strategy on PROSPERO 2020 database obtaining the following registration code: CRD42020180856. The outcomes collected were: ORR, PFS, OS, treatment-related advent events (TRAEs) and discontinuation rates. Only data on studies enrolling patients $\geq 18$ years were included. No sex restriction was considered. The trial selection was initially performed by two authors (AG and FP), who independently screened and selected abstracts and titles according to the aforementioned exclusion and inclusion criteria. Disagreements were discussed and finally solved with a third author (AR).

\section{Selection, data extraction, and quality assessment of the included studies}

Two authors independently extracted data (AG and FP). Disagreements were discussed and finally solved with a third author (AR). They reported information about the included studies (trial name, drug regimens, sample size, outcomes). Every outcome was carried out according to PD-L1 expression level (<1\%, 1-49\%, $\geq 50 \%)$ and collected in a pre-planned repository sheet. In the case of articles with follow-up over time, we decided to include the most updated and methodologically valid one. To determine the overall quality of the eligible randomized trials, we conducted a study quality assessment using the Jadad score calculation for every study following the criteria reported in the Cochrane Handbook for Systematic Reviews of Interventions (19) including: allocation concealment; blinding of participants, personnel and outcome assessors; incomplete outcome data; sequence generation; elective outcomes reporting; other sources of bias. For each study, we defined "Yes" as at low risk of bias and "No" as at high risk of bias. We defined also "unclear" if there were insufficient data for a precise judgment. The risk of selective outcome reporting bias was also evaluated by two independent reviewers (AG and $\mathrm{VG}$ ) and disagreements were solved by consensus.

\section{Statistical analysis}

RevMan ver 5.3 and Comprehensive Meta-analysis version 2.2.064 were used to perform the statistical analysis. The considered outcomes of interests for both direct and indirect comparisons were ORR, PFS, OS, TRAEs and discontinuation rate. We used hazard ratios (HRs) to assess the association for PFS and OS, with a 95\% confidence interval (CI). For all other outcomes (ORR, TRAEs, discontinuation rate) we calculated the total number of events over the total patients randomized in each group, thus using the Risk Ratios (RRs) as a measure of association. In the first phase of the study, using meta-analysis techniques, we performed a direct comparison including all the RCTs evaluating the efficacy of intervention arms (IO or $\mathrm{IO}+$ CT or Combo IO) versus standard CT, by calculating the logarithm of the HR (logHRs) or RR (logRRs) and its relative standard error (SE) for all the RCTs included in this phase. Afterward, we calculated pooled data of every comparison.

We took final estimates from direct comparisons in order to obtain pooled estimates of $\mathrm{HR}$ and RR needed for the indirect comparison. The method used for the indirect comparison was that described by Bucher and Glenny, extended to calculate the HR (20). We used this method for its ability to maintain the randomization advantage of 
each trial providing an estimate of the comparison between treatments. For example, assuming that $\mathrm{IO}_{\mathrm{st}}$ is the estimate of the direct comparison between IO and standard CT, and that $\mathrm{IO}+\mathrm{C}_{\mathrm{st}}$ is the estimate of the direct comparison between $\mathrm{IO}+\mathrm{CT}$ and $\mathrm{CT}$, then the estimate of the indirect comparison between IO + CT and IO may be calculated as follows: $\mathrm{IO}+\mathrm{CT} / \mathrm{IO} \_$indirect $(\log \mathrm{HR}$ or $\log \mathrm{RR})=\mathrm{IO}+$ $\mathrm{CT}_{\mathrm{st}}(\log \mathrm{HR}$ or $\log \mathrm{RR})-\mathrm{IO}_{\mathrm{st}}(\log \mathrm{HR}$ or $\log \mathrm{RR})$.

The variance can be obtained with the following computation: $\operatorname{Var}\left(\log \mathrm{IO}+\mathrm{CT} / \mathrm{IO} \_\right.$indirect $)=\operatorname{Var}(\log$ $\left.\mathrm{IO}+\mathrm{CT}_{\mathrm{st}}\right)+\operatorname{Var}\left(\log \mathrm{IO}_{\mathrm{st}}\right)(21)$. For the $\mathrm{IO}+\mathrm{CT}$ versus IO comparison, heterogeneity between studies was tested using the Cochran Q statistic and quantified by the $\mathrm{I}^{2}$ value. If the $\mathrm{I}^{2}$ value was higher than $50 \%$, with a high risk of heterogeneity, we performed the meta-analysis using the random effect-based model according to Der Simonian and Laird; for the $\mathrm{I}^{2}$ lower than $50 \%$ we used the fixedeffect based Mantel-Haenszel model (22). In our example indirect meta-analysis outcomes with $\mathrm{HR}<1$ would suggest a better efficacy of IO + CT, whereas toxicity outcome with $\mathrm{RR}<1$, would suggest a better toxicity profile for singleagent IO regimen. The same statistical considerations were used to evaluate Combo IO versus IO and Combo IO versus IO + CT comparisons. When applicable, subgroup analyses were performed according to PD-L1 expression level $(<1 \%, 1-49 \%, \geq 50 \%)$ and ICIs categories (PD-1 versus PD-L1 inhibitors). As regards the between studies bias, we performed a publication bias test using Egger's test providing the Funnel Plot for asymmetry. The manuscript was done and reported according to the PRISMAguidelines for reporting on systematic reviews (23). P values were considered statistically significant if $\mathrm{P}<0.05$.

\section{Results}

The literature search identified a total of 791 records; 701 records were excluded because of systematic reviews or meta-analyses, retrospective or phase I/II studies, not human studies, consensus, or guidelines. A total of 90 trials were assessed for eligibility and 76 were excluded because no drugs of interest or data about the principal outcomes of our indirect comparison (ORR, PFS, OS, TRAEs, discontinuation rate) were reported. Finally, a total of fourteen studies (11,656 patients) met our inclusion/ exclusion criteria and were included within the indirect comparisons (Figure S2). The main baseline characteristics and the outcomes measures of each included trial are reported in Tables 1 and 2, respectively.

\section{Direct comparisons}

\section{Single-agent IO versus CT}

Six RCTs enrolling 4,387 patients evaluated single-agent IO (two for pembrolizumab, one for atezolizumab, one for nivolumab, one for cemiplimab and one for durvalumab) versus CT in advanced NSCLC. In the PD-L1 $\geq 50 \%$ subgroup, pooled results showed clear statistically significant differences in terms of ORR (RR 1.39, 95\% CI: 1.23-1.57), PFS (HR 0.65, 95\% CI: 0.53-0.79) and OS (HR 0.67, 95\% CI: 0.61-0.74) favoring the single-agent IO arm (Figure S3). Only one trial was included for the PD-L1 1-49\% subgroup, showing no benefit in terms of OS (HR 0.91, 95\% CI: 0.76-1.09) (Figure S4A). Likewise, as for the PDL1 $<1 \%$ subgroup, no clear OS benefit in favor of singleagent IO over CT was shown (HR 0.95, 95\% CI: 0.63-1.43) (Figure S4B). No results for ORR and PFS were available. Single-agent IO was associated with a significantly reduced risk of TRAEs over CT alone (RR 0.44, 95\% CI: 0.36-0.52), with no significant differences in terms of discontinuation rate (RR 0.97, 95\% CI: 0.62-1.53) (Figure S5).

\section{$\mathrm{IO}+\mathrm{CT}$ versus $\mathrm{CT}$}

Eight RCTs enrolling 5,421 patients evaluated the combination of IO + CT (two for pembrolizumab, four for atezolizumab, one for nivolumab and one for nivolumab plus ipilimumab) versus CT in advanced NSCLC. In the subgroup of PD-L1 $\geq 50 \%$, the addition of IO to standard CT produced a significant advantage in terms of ORR (RR 1.91, 95\% CI: 1.56-2.34), PFS (HR 0.44, 95\% CI: $0.37-$ 0.52 ) and OS (HR 0.65, 95\% CI: 0.54-0.77) (Figure S6). Our results showed a consistent superiority of the combination regimens even in the PD-L1 1-49\% and PDL1 $<1 \%$ subgroups, including a significant increase of ORR (RR 1.34, 95\% CI: 1.12-1.60; RR 1.55, 95\% CI: 1.33-1.81); PFS (HR 0.63, 95\% CI: 0.56-0.71; HR 0.71, 95\% CI: 0.64 0.78 ); and OS (HR 0.75, 95\% CI: 0.64-0.87; HR 0.75, 95\% CI: $0.67-0.83$ ), respectively (Figures S7,S8). The addition of IO to CT regimens produced a significant increase of TRAEs (RR 1.21, 95\% CI: 1.15-1.28) and treatment discontinuation (RR 1.78, 95\% CI: 1.42-2.23) (Figure S9).

\section{Combo IO versus CT}

Only two RCTs enrolling 1910 patients evaluated PD-1/ PD-L1 plus CTLA-4 combinations (one for nivolumab + ipilimumab, one for durvalumab + tremelimumab) versus CT. In the PD-L $1 \geq 50 \%$ subgroup, a not significant increase of both ORR (RR 1.25, 95\% CI: 0.98-1.60) and 
Table 1 Main characteristics and safety outcomes of the selected studies

\begin{tabular}{|c|c|c|c|c|}
\hline Trial & Treatment arm & Number of patients & TRAEs G3-5, $\mathrm{n}$ & Discontinuation, $\mathrm{n}$ \\
\hline KEYNOTE-042 & Pembrolizumab vs. platinum-based CT & 637 vs. 637 & $117 / 636$ vs. $253 / 615$ & $62 / 636$ vs. $59 / 615$ \\
\hline IMpower-110 & Atezolizumab vs. platinum-based CT & 286 vs. 286 & $37 / 286$ vs. $117 / 263$ & $18 / 286$ vs. $43 / 263$ \\
\hline CheckMate-227 Part 1 & Nivolumab vs. platinum-based CT & 396 vs. 397 & $76 / 391$ vs. $141 / 387$ & $28 / 391$ vs. $11 / 387$ \\
\hline EMPOWER-Lung 1 & Cemiplimab vs. platinum-based CT & 355 vs. 342 & $50 / 355$ vs. $134 / 342$ & $52 / 355$ vs. $39 / 342$ \\
\hline KEYNOTE-189 & $\begin{array}{l}\text { Pembrolizumab + platinum-pemetrexed vs. } \\
\text { platinum-pemetrexed }\end{array}$ & 410 vs. 206 & $196 / 405$ vs. $80 / 202$ & $85 / 405$ vs. $17 / 202$ \\
\hline KEYNOTE-407 & $\begin{array}{l}\text { Pembrolizumab + carboplatin + (nab-) } \\
\text { paclitaxel vs. carboplatin + (nab-)paclitaxel }\end{array}$ & 278 vs. 281 & $152 / 278$ vs. $154 / 280$ & $50 / 278$ vs. $20 / 280$ \\
\hline IMpower-130 & $\begin{array}{l}\text { Atezolizumab + carboplatin + nab-paclitaxel } \\
\text { vs. carboplatin + nab-paclitaxel }\end{array}$ & 483 vs. 240 & $354 / 473$ vs. $141 / 232$ & $125 / 473$ vs. $51 / 232$ \\
\hline IMpower-132 & $\begin{array}{l}\text { Atezolizumab + platinum-pemetrexed vs. } \\
\text { platinum-pemetrexed }\end{array}$ & 292 vs. 286 & $167 / 291$ vs. $114 / 274$ & $69 / 291$ vs. $48 / 274$ \\
\hline IMpower-131 & $\begin{array}{l}\text { Atezolizumab + carboplatin + nab-paclitaxel } \\
\text { vs. carboplatin + nab-paclitaxel }\end{array}$ & 343 vs. 340 & $231 / 334$ vs. $195 / 334$ & $102 / 334$ vs. $58 / 334$ \\
\hline CheckMate-227 Part 2 & $\begin{array}{l}\text { Nivolumab + platinum-based CT vs. } \\
\text { platinum-based CT }\end{array}$ & 377 vs. 378 & $45 / 375$ vs. $35 / 371$ & $30 / 375$ vs. $11 / 371$ \\
\hline
\end{tabular}

TRAEs, treatment-related adverse events; G, grade; n, number.

PFS (HR 0.62, 95\% CI: 0.30-1.28), along with a significant OS gain observed in favor of the Combo IO arm (HR 0.72, 95\% CI: 0.60-0.86) (Figure S10). No OS differences have been detected in the subgroup of PD-L1 1-49\% (HR 0.94, 95\% CI: 0.75-1.18) (Figure S11), while a significant benefit in terms of both PFS (HR 0.75, 95\% CI: 0.59-0.96) and OS (HR 0.65, 95\% CI: 0.53-0.80) favoring the Combo IO has been reported in those patients with PD-L1 expression $<1 \%$ (Figure S12). As a counterpart, an increased percentage of patients receiving Combo IO discontinued the treatment (HR 1.79, 95\% CI: 1.40-2.30) as compared to standard CT, although no significant TRAEs differences have been registered between the two treatments strategies (HR 0.80,
95\% CI: 0.60-1.06) (Figure S13).

\section{Indirect comparisons}

We used the meta-analytic technique to perform an indirect comparison between the three different immune-based strategies on efficacy endpoints according to different PDL1 expression levels, and safety endpoint in the overall population.

Efficacy PD-L1 $\geq 50 \%$

The IO + CT combination was associated with an increased response rate over both single-agent IO (RR 1.37, 95\% CI: 


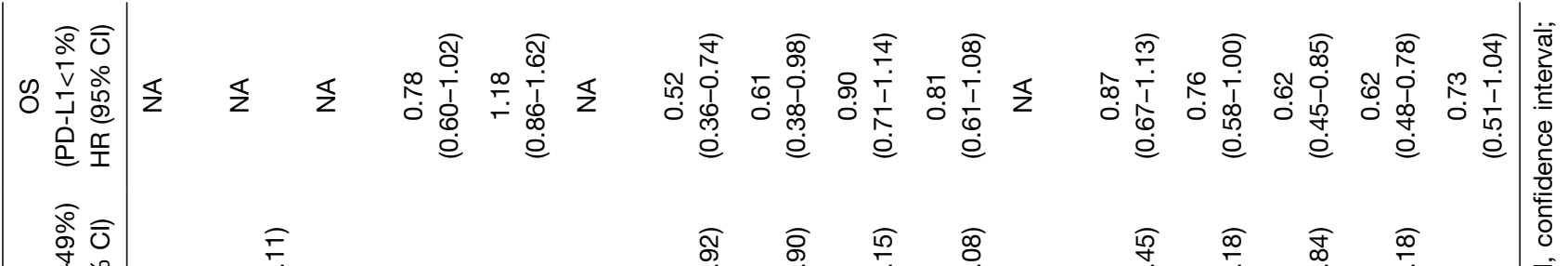

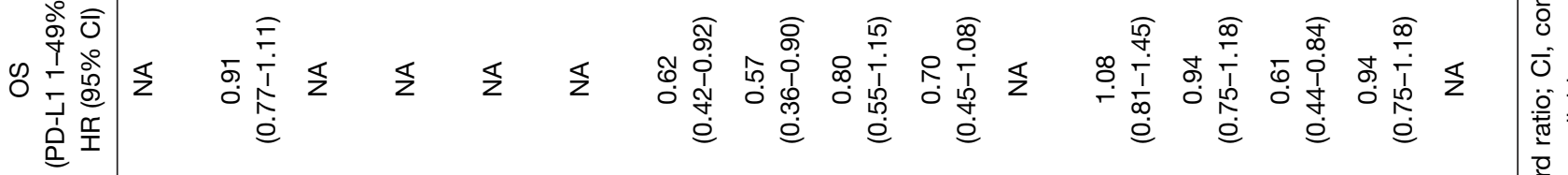

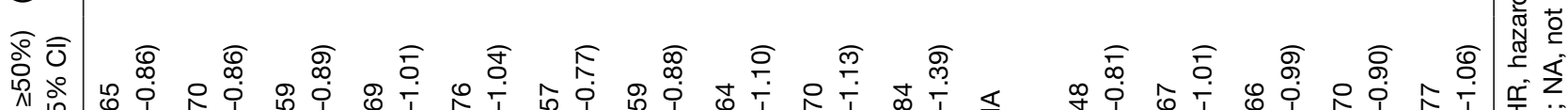

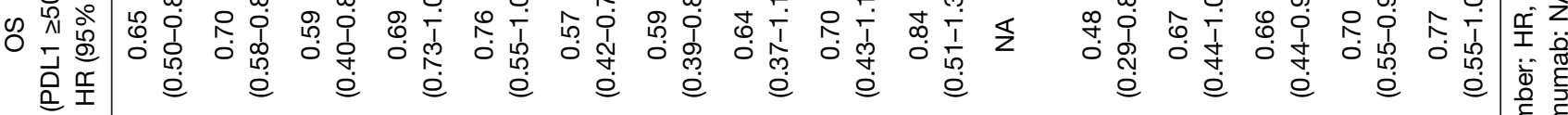

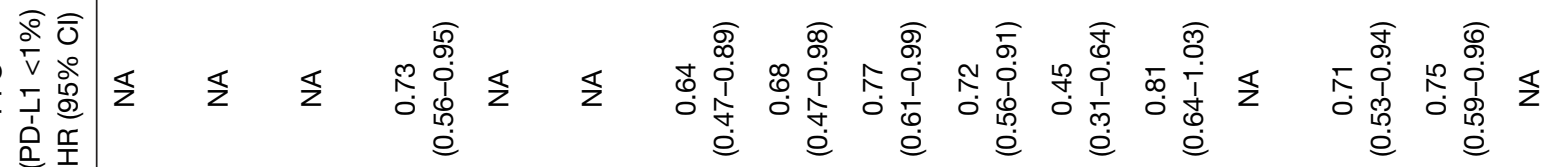

๙ัे

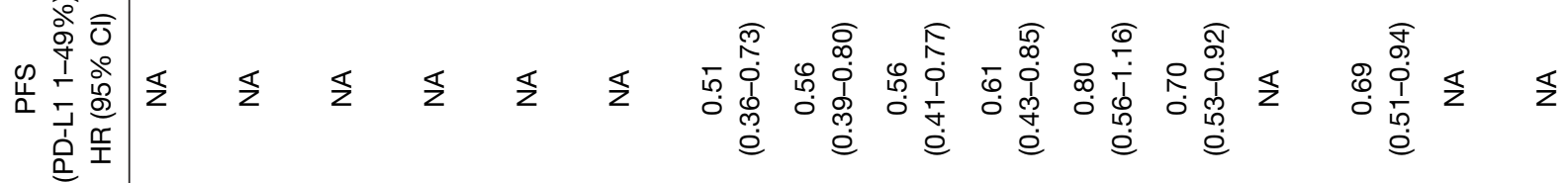

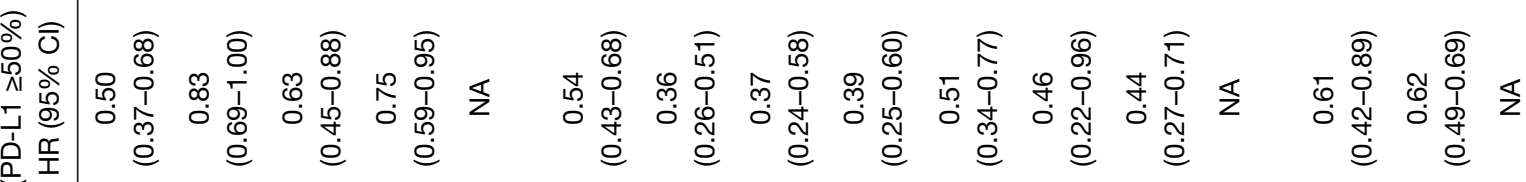

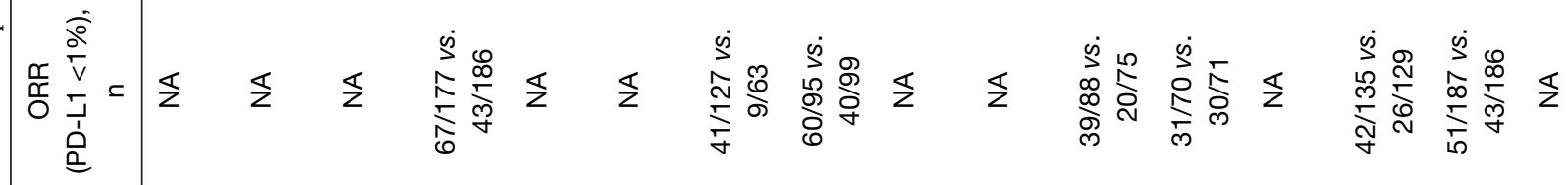

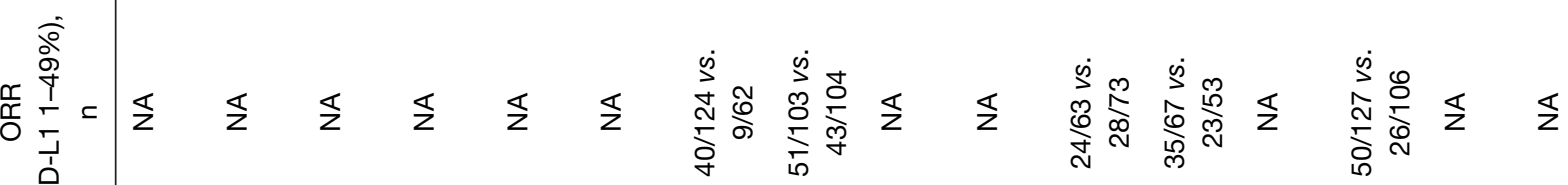

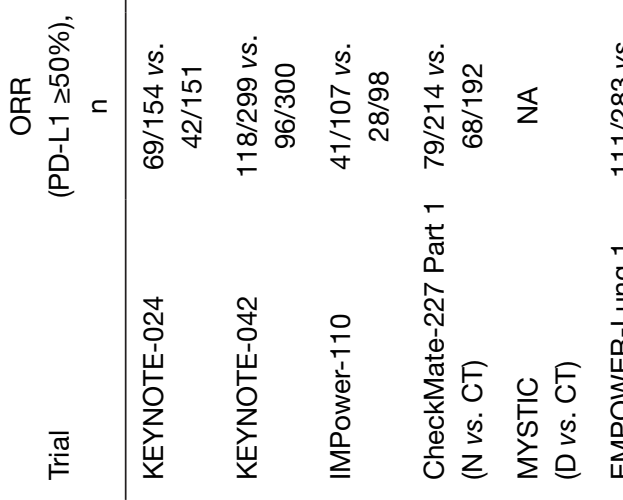

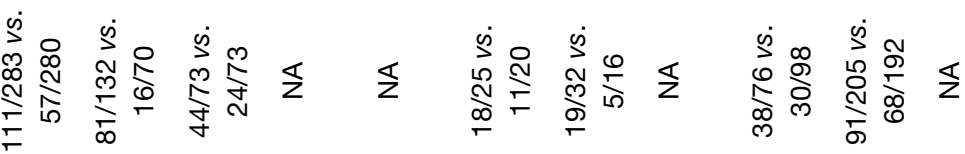

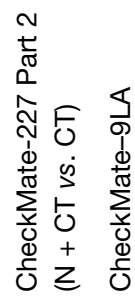

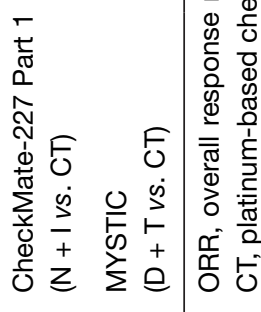




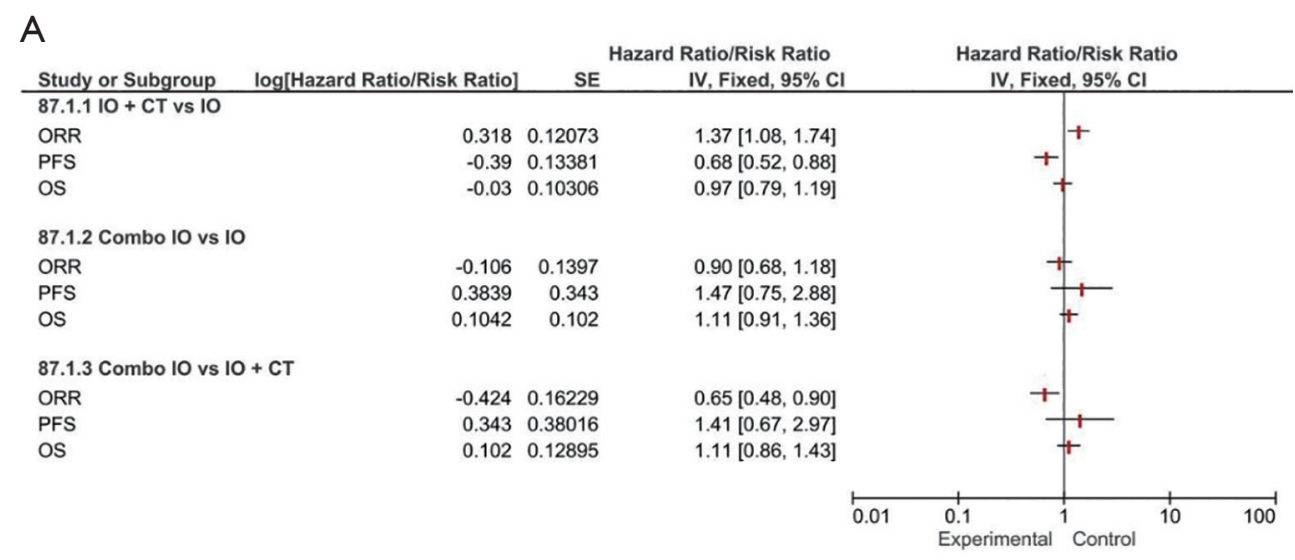

B

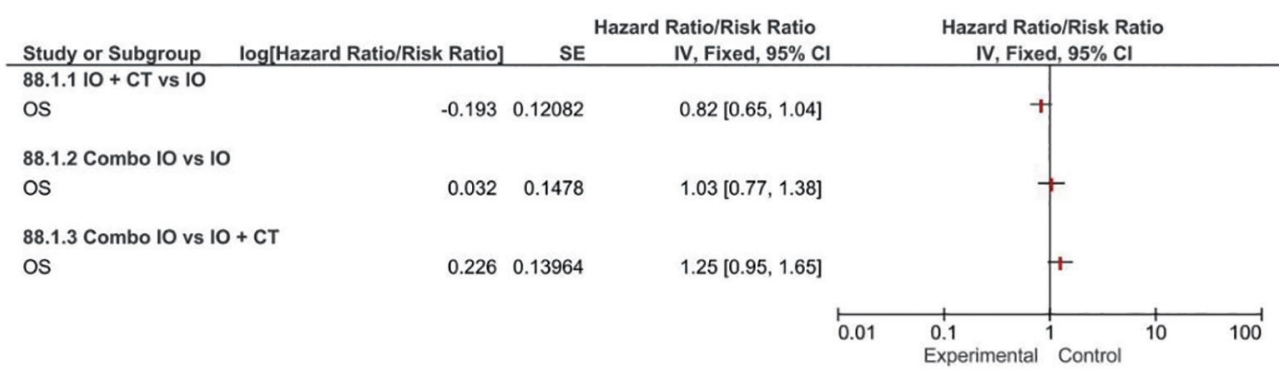

C

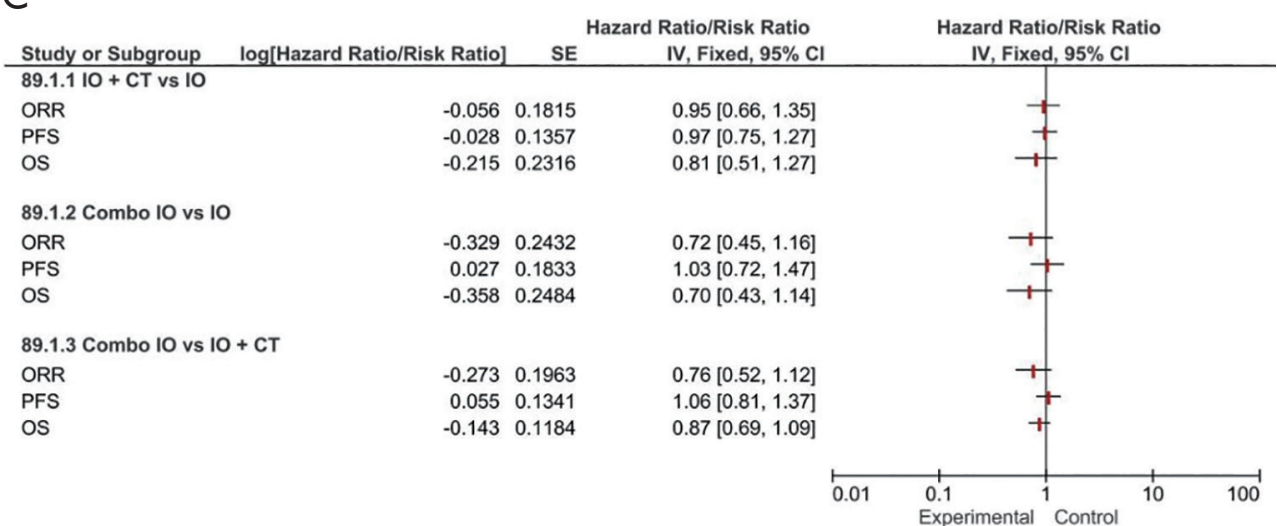

Figure 1 Forest plots for efficacy endpoints indirect comparisons among combination regimens (IO, IO + CT, Combo IO) according to PD-L1 expression: PD-L1 250\% (A); PD-L1 1-49\% (B); PD-L1 <1\% (C). IO, single-agent immunotherapy; IO + CT, immunotherapy plus standard platinum-based chemotherapy; Combo IO, immunotherapy combination regimens; ORR, overall response rate; PFS, progressionfree survival; OS, overall survival.

1.08-1.74) and Combo IO (RR 0.65, 95\% CI: 0.48-0.90). Interestingly, IO + CT combination was associated with a PFS benefit when compared to single-agent IO (HR 0.68, 95\% CI: 0.52-0.88). No other significant differences in terms of PFS and OS were observed among the three treatment regimens (Figure $1 A$ ).

\section{Efficacy PD-L1 1-49\%}

Only OS data were available from direct comparison to perform our analysis. Finally, our results showed no significant OS differences between the three different immune-based treatment strategies $(\mathrm{IO}+\mathrm{CT}$ versus $\mathrm{IO}$ or Combo $\mathrm{IO}$ versus $\mathrm{IO}$ or Combo IO versus IO + CT) (Figure 1B). 


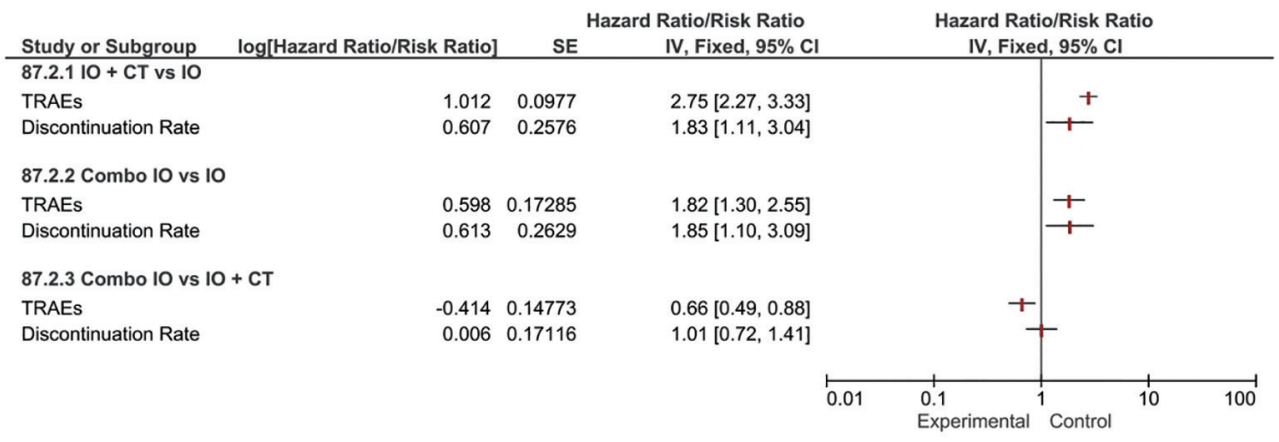

Figure 2 Forest plots for safety endpoints indirect comparisons among all the combination regimens (IO, IO + CT, Combo IO). IO, singleagent immunotherapy; IO + CT, immunotherapy plus standard platinum-based chemotherapy; Combo IO, immunotherapy combination regimens; TRAEs, treatment-related adverse events.

\section{Efficacy PD-L1 $<1 \%$}

According to the available data, our pooled results interestingly showed a not significant increase of ORR in favor of IO + CT compared to both single-agent and Combo IO strategy, with no differences in terms of both PFS and OS (Figure 1C). No activity and survival differences between single-agent and Combo IO have been observed in this subgroup.

\section{Safety}

Indirect comparisons suggested that combination strategies (Combo IO or IO + CT) both overcame single-agent IO in terms of TRAEs incidence risks (RR 1.82, 95\% CI: 1.30-2.55 and RR 2.75, 95\% CI: 2.27-3.33, respectively), resulting in slightly higher risk (RR 1.85, 95\% CI: 1.10-3.09 and RR 1.83 , 95\% CI: 1.11-3.04, respectively) of treatment discontinuation. Indirect comparison between the two different combination regimens revealed that Combo IO produced a substantially lower rate of TRAEs (RR 0.66, 95\% CI: 0.49-0.88) compared to IO + CT, however not significantly influencing the discontinuation rate of any treatment component (Figure 2).

\section{Subgroup analysis}

We performed a subgroup analysis to separately assess the specific contribution of PD-1 and PD-L1 inhibitors to each immune-based treatment strategy, across the different PDL1 selected subgroups.

As concerns the role of PD-1 inhibitors in the PD-L1 $\geq 50 \%$ subpopulation, our subgroup analysis confirmed that the addition of an anti-PD-1 to CT provided a remarkable contribution in terms of response rate over both IO monotherapy (RR 1.44, 95\% CI: 1.01-2.04) and
Combo IO (RR 0.62, 95\% CI: 0.44-0.87). Interestingly, a substantial advantage in terms of PFS (HR 0.66, 95\% CI: $0.48-0.92$ ) was observed in favor of PD-1 inhibitors when indirectly comparing $\mathrm{IO}+\mathrm{CT}$ versus IO, however with no additionally significant OS differences among all the different treatment strategies (Figure 3). Likewise, the addition of PD-L1 inhibitors to standard CT was associated with a slight advantage over single-agent IO in terms of PFS (HR 0.71, 95\% CI: 0.47-1.08) that did not eventually translate into an activity and OS benefit. No further OS differences have been observed among the remaining subgroups albeit considering that full indirect comparisons could not be conducted because of missing ORR and PFS data (Figure 4). Of note, in the PD-L1 1-49\% subgroup, the use of anti-PD-1 agents in association with CT led to a clinically and statistically significant gain in OS over IO monotherapy (HR 0.66; 95\% CI: 0.49-0.88) and Combo IO (HR 1.57, 95\% CI: 1.14-2.15) (Figure 3B); while considering PD-L1 agents in this subgroup, data were not available in this subpopulation. About the PD-L1 negative patients, PD-1 inhibitors as Combo IO seemed to yield less ORR (RR 0.72, 95\% CI: 0.48-1.07) than as IO + CT, however with no additional differences in survival; likewise, no further OS differences between single-agent IO and both combination approaches have been observed for both PD-1 and PD-L1 agents (Figures 3C,4B).

Noteworthy, when evaluating the pooled safety profile of both anti-PD-1 and anti-PD-L1 agents, our results showed that patients receiving the association of IO plus CT seem to experience a higher risk of adverse events (RR 0.92, 95\% CI: $0.71-1.13$ and RR $1.23,95 \%$ CI: $0.82-1.64$, respectively) and treatment discontinuation (RR 0.67 , 95\% CI: $0.26-1.08$ and RR 1.09, 95\% CI: 0.69-1.49, 


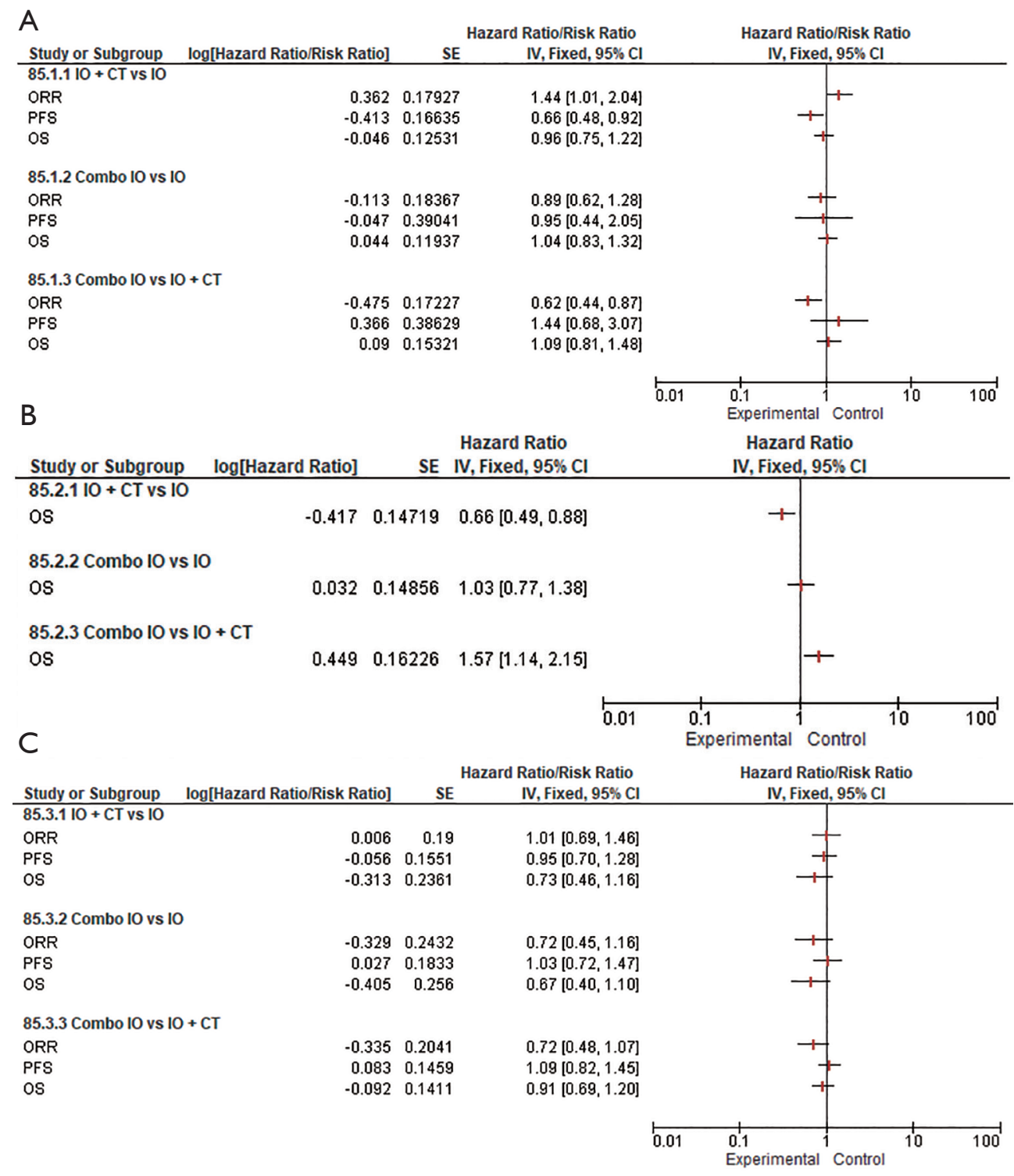

Figure 3 Forest plots for efficacy endpoints indirect comparisons among combination regimens (IO, IO + CT, Combo IO) for PD-1 agents according to PD-L1 expression: PD-L1 250\% (A); PD-L1 1-49\% (B); PD-L1 <1\% (C). IO, single-agent immunotherapy; IO + CT, immunotherapy plus standard platinum-based doublet; Combo IO, immunotherapy combination regimens; ORR, overall response rate; PFS, progression-free survival; OS, overall survival.

respectively) when indirectly compared to single-agent IO. Likewise, as expected the dual IO blockade led to a heightened toxicity risk over single-agent IO for both PD-1 and PD-L1 inhibitors. Furthermore, when indirectly considering the combination strategies, both PD-1 and PD-L1 inhibitors in the Combo IO approach led to a lower risk of TRAEs over IO + CT (RR $-0.26,95 \%$ CI: -0.45 to -0.07 and RR $-0.59,95 \%$ CI: -0.83 to -0.35 , respectively), however with no significant differences in the discontinuation rate (Figure $5 A, B$ ).

\section{Risk of bias assessment}

Publication bias test is necessary for a meta-analysis including at least 3 studies. In our analysis, Egger's test was calculated for every outcome showing no statistical 


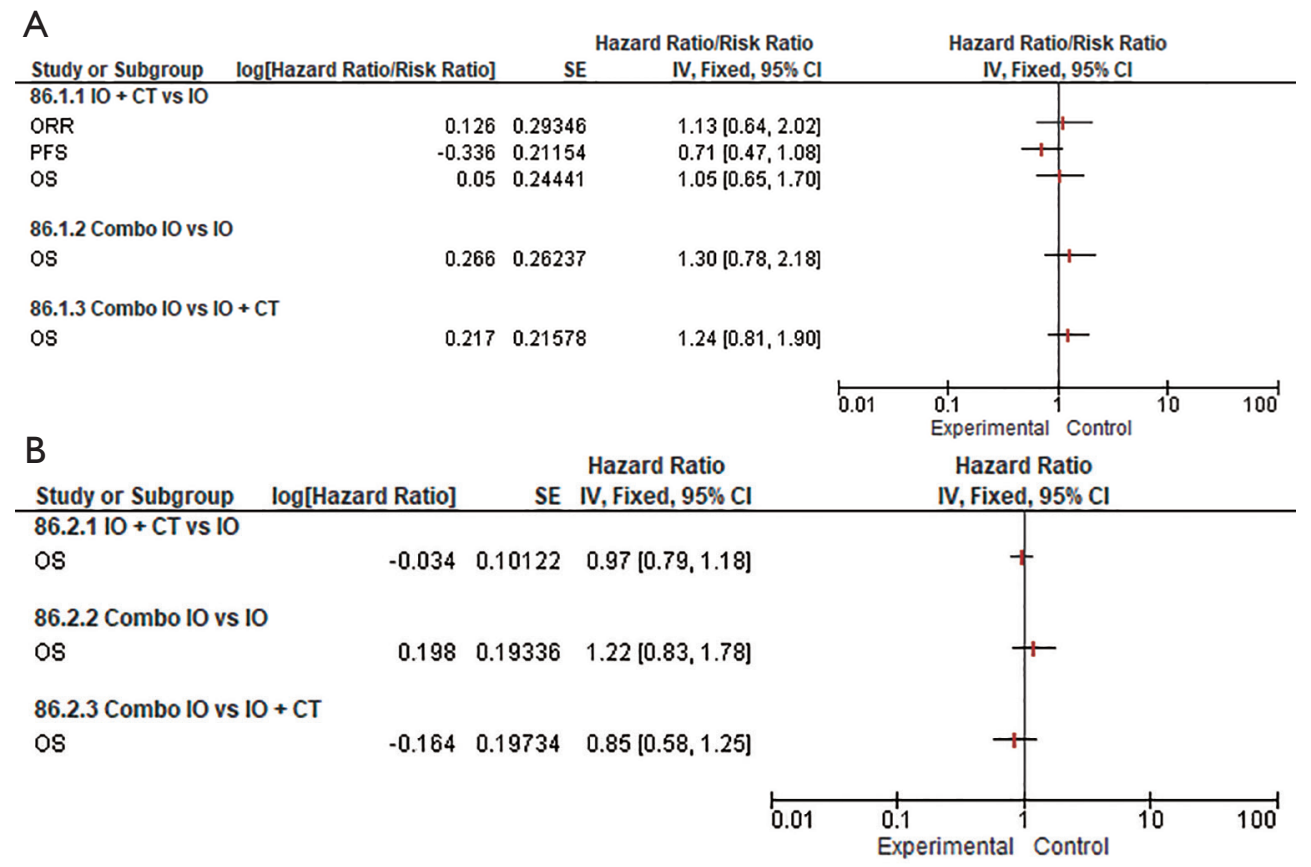

Figure 4 Forest plots for efficacy endpoints indirect comparisons among combination regimens (single IO agent, IO + CT, Combo IO) for PD-L1 agents according to PD-L1 expression: PD-L1 250\% (A); PD-L1 <1\% (B). No data available for PD-L1 1-49\% subgroup. IO, single-agent immunotherapy; IO + CT, immunotherapy plus standard platinum-based doublet; Combo IO, immunotherapy combination regimens; ORR, overall response rate; PFS, progression-free survival; OS, overall survival.

significance (Figure S14). The overall quality assessment was evaluated according to the CONSORT checklist statement. We reported an average good quality of all trials. Some problems related to "random sequence generation" and "allocation concealment" (selection bias) domains, because all the selected studies were open-label and full papers, were not available at all at the time of this report (Figure S15).

\section{Discussion}

The results of this indirect metanalysis demonstrated that the $\mathrm{IO}+\mathrm{CT}$ combination has the best activity profile among the three different IO-based treatment strategies currently available for the first-line treatment of patients with PD-L $1 \geq 50 \%$ advanced NSCLC, leading to a significant increase of the ORR which did not translate into a relevant improvement of patients' survival. Similarly, no survival differences between the three different IO-based strategies have been observed in the PD-L1-low subgroup. As regards patients with negative PD-L1 expression, no significant differences in terms of activity and efficacy profile have been detected between the $\mathrm{IO}+\mathrm{CT}$ and the dual checkpoint blockade. Considering that the majority of IO-based treatment regimens currently approved for clinical use include PD-1 agents, we decided to separately analyze clinical trials evaluating PD-1 and PD-L1 agents, to provide a more precise estimation of potential activity/ efficacy differences, which may influence our current practice. The results of our subgroup analysis confirmed that in PD-L1-high patients the combination of PD-1 agents and CT is associated with a significant increase of activity (ORR and PFS) as compared to both single-agent PD-1 and PD-1/PD-L1 + CTLA-4 blockade. Similarly, a significant increase of the ORR in favor of the $\mathrm{IO}+\mathrm{CT}$ combination has been observed also within the PD-L1 negative subgroups. Importantly, PD-1 + CT combinations led to a significant improvement of OS as compared to both single-agent PD-1 and PD-1/PD-L1 + CTLA-4 blockade in PD-L1-low subgroup, emerging as the most effective strategy for the treatment of these patients' subset in our current practice. As expected, both $\mathrm{IO}+\mathrm{CT}$ and Combo IO strategies produced a significant increase of grade 3-5 TRAEs as compared to single-agent IO, while Combo IO 


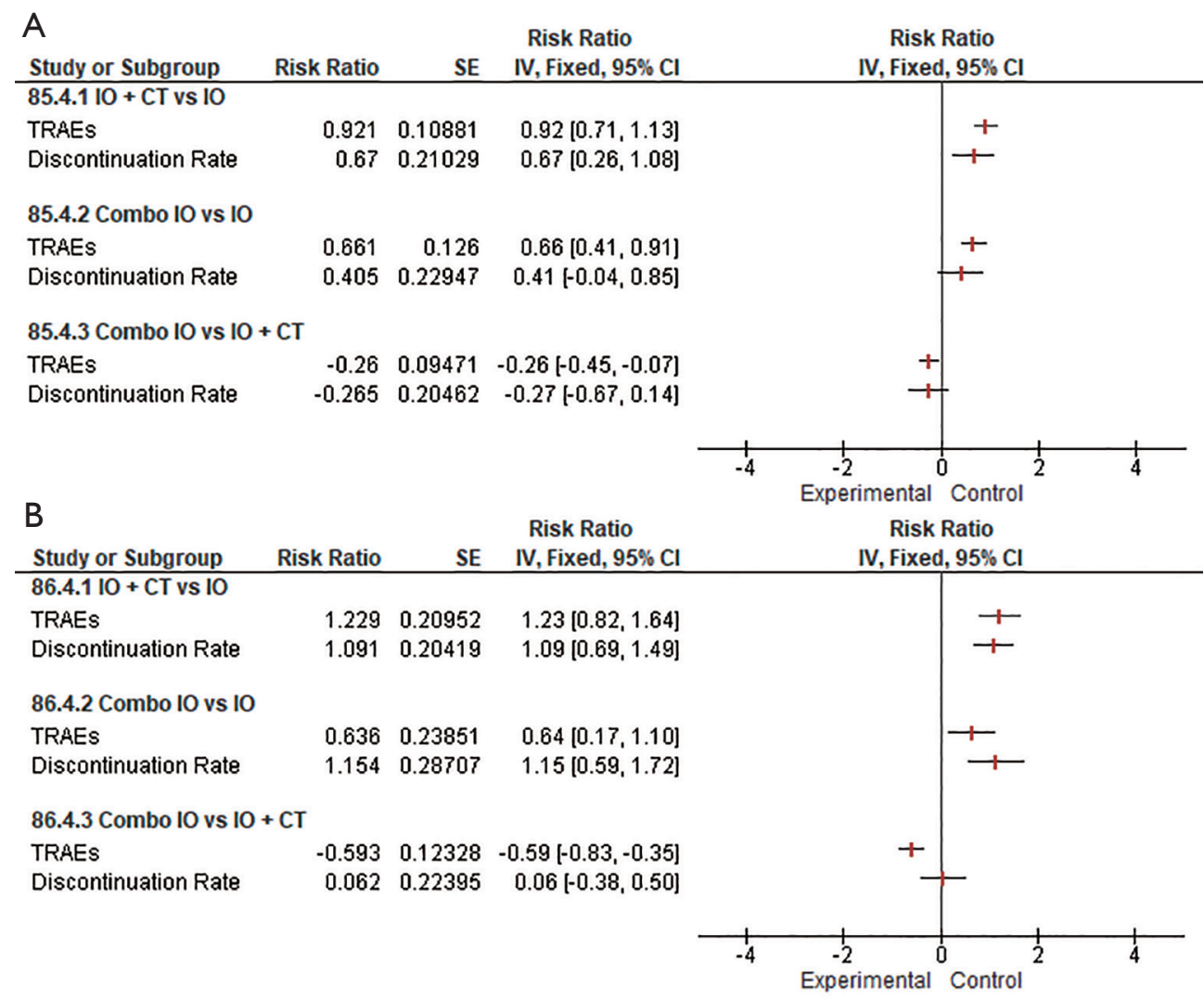

Figure 5 Forest plots for safety endpoints indirect comparisons among combination regimens (IO, IO + CT, Combo IO) according to different IO agents: anti-PD-1 (A); anti-PD-L1 (B). IO, single-agent immunotherapy; IO + CT, immunotherapy plus standard platinumbased doublet; Combo IO, immunotherapy combination regimens; TRAEs, treatment-related adverse events.

was associated with a lower incidence of serious TRAEs as compared to $\mathrm{IO}+\mathrm{CT}$ in the overall PD-L1 unselected population.

Translating the results of this analysis to our current practice, single-agent pembrolizumab is confirmed to be a valid option for the treatment of $\mathrm{PD}-\mathrm{L} 1 \geq 50 \%$ advanced NSCLC, since it has been associated with the best efficacy/ tolerability profile as compared to the other combination strategies. Indeed, both IO + CT and the dual checkpoint blockade did not outperform single-agent IO survival outcomes, resulting to double the grade 3-5 TRAEs. These results appeared to confirm the latest findings from the KEYNOTE-598 and EMPOWER-Lung 1 trials, in which patients with high PD-L1 expression did benefit the most from a single-agent IO treatment in the first-line setting $(12,24)$. However, considering its best anti-tumor activity, the association of pembrolizumab with CT could be preferred in those patients with high disease burden and/or cancer-related symptoms to whom a rapid tumor volume decrease would be desirable, and in any case to prevent the phenomenon of hyperprogressive disease which may conversely occur in those patients receiving ICI alone or dual ICI blockade. Since the current evidence does not support the use of nivolumab plus ipilimumab combination in metastatic NSCLC patients with both high ( $\geq 50 \%)$ and low (1-49\%) PD-L1 expression, however the most promising role for this combination is emerging in PD-L1 negative NSCLC. Indeed, considering the similar efficacy outcomes along with the lower grade 3-5 TRAEs incidence reported by our analysis, the dual checkpoint blockade could be considered as a valid alternative option to the IO + CT combination for the clinical management of this subgroup of patients. In this regard, the CheckMate-227 trial also showed that the nivolumab-ipilimumab arm was associated with a longer duration of response (18 versus 8.3 months) along with a not significant trend toward an increased 2-year 
OS (40.4\% versus $34.7 \%)$ as compared to nivolumab-CT arm, in the subgroup of PD-L1 negative patients (10). However, it should be noted that the nivolumab-CT related survival within the Checkmate-227 trial underperformed the pembrolizumab-CT one within the KEYNOTE 021/189/407 pooled analysis (25). Therefore, the role of nivolumab-ipilimumab in this subgroup of NSCLC patients would require further investigation in dedicated studies including the current standard of care, pembrolizumab-CT, as the control arm. Although not included in this indirect comparison, the incidence of G3-5 immune-related TRAEs was about $23 \%$ with the alternative dosing regimen of nivolumab $(3 \mathrm{mg} / \mathrm{kg})$ plus ipilimumab $(1 \mathrm{mg} / \mathrm{kg})$ used in the CheckMate-227 trial (10) compared to $10 \%$ reported in the pembrolizumab-CT studies (13), representing another relevant point to be considered during the selection of the best upfront treatment. More recently, the results of the CheckMate 9LA trial revealed that the association of nivolumab-ipilimumab combination with a short course (2 cycles) of CT significantly improved NSCLC patients' OS when directly compared to CT alone (HR 0.66; 95\% CI, 0.55-0.80) regardless of PD-L1 expression levels, emerging as an additional potential upfront IO-based treatment option requiring confirmation within longer follow-up (17).

Even if performing multiple indirect comparisons of first-line IO-based combinations across PD-L1 selected populations, however this study had several limitations. First, these results should always be interpreted with caution since they are based on indirect comparisons not at individual patient-level. Secondly, indirect comparisons were not computed for every single outcome across all the PD-L1 selected studies and/or according to the histological subtype because of lacking data. Thirdly, when evaluating the role of dual ICIs blockade in association with CT, as recently emerging from the CheckMate 9LA trial (17), the contribution of a shorter CT course as a significant source of heterogeneity when compared to other CT backbones of the other trials must be considered; moreover, we could not include in the final analysis the latest findings from the KEYNOTE-598 trial (24) because of the absence of CT as common control arm. Lastly, the heterogeneity of different assays used in the selected studies assessing the PD-L1 expression level could influence the prediction of the immune response.

In conclusion, the results of this indirect comparison meta-analysis suggested that PD1+CTLA-4 inhibitors combination has a current limited role in the treatment of patients with PD-L1 high ( $\geq 50 \%)$ and/or low (1-49\%) metastatic NSCLC, while emerging as a potentially effective and tolerable option in particular subgroups of patients with PD-L1 negative disease. However, longer follow-up and further prospective investigation are needed to definitively establish whether there will be a place for the PD-1/CTLA-4 inhibitors combination in the first-line treatment of advanced non-oncogene addicted NSCLC.

\section{Acknowledgments}

Francesco Passiglia, Valerio Gristina and Maria La Mantia contributed to the current work under the Doctoral Programme in Experimental Oncology and Surgery, University of Palermo. The authors thank Dr. Chiara Drago for the English language revision.

Funding: None.

\section{Footnote}

Reporting Checklist: The authors have completed the PRISMA reporting checklist. Available at https://dx.doi. org/10.21037/tlcr-21-52

Peer Review File: Available at https://dx.doi.org/10.21037/ tlcr-21-52

Conflicts of Interest: All authors have completed the ICMJE uniform disclosure form (available at https://dx.doi. org/10.21037/tlcr-21-52). FP received grant consultancies by MSD and Astrazeneca. Prof. AR reported personal fees from Bristol, Pfizer, Bayer, Kyowa Kirin, Ambrosetti for advisory board activity; speaker honorarium from Roche Diagnostics. The authors have no other conflicts of interest to declare.

Ethical Statement: The authors are accountable for all aspects of the work in ensuring that questions related to the accuracy or integrity of any part of the work are appropriately investigated and resolved.

Open Access Statement: This is an Open Access article distributed in accordance with the Creative Commons Attribution-NonCommercial-NoDerivs 4.0 International License (CC BY-NC-ND 4.0), which permits the noncommercial replication and distribution of the article with the strict proviso that no changes or edits are made and the original work is properly cited (including links to both the formal publication through the relevant DOI and the license). 
See: https://creativecommons.org/licenses/by-nc-nd/4.0/.

\section{References}

1. Garon EB, Hellmann MD, Rizvi NA, et al. Five-Year Overall Survival for Patients With Advanced Non-SmallCell Lung Cancer Treated With Pembrolizumab: Results From the Phase I KEYNOTE-001 Study. J Clin Oncol 2019;37:2518-27.

2. Mok TSK, Wu YL, Kudaba I, et al. Pembrolizumab versus chemotherapy for previously untreated, PD-L1expressing, locally advanced or metastatic non-small-cell lung cancer (KEYNOTE-042): a randomised, open-label, controlled, phase 3 trial. Lancet 2019;393:1819-30.

3. Paz-Ares LG, Luft A, Tafreshi A, et al. Phase 3 study of carboplatin-paclitaxel/nab-paclitaxel (Chemo) with or without pembrolizumab (Pembro) for patients (Pts) with metastatic squamous $(\mathrm{Sq})$ non-small cell lung cancer (NSCLC). J Clin Oncol 2018;36:105.

4. Socinski MA, Jotte RM, Cappuzzo F, et al. Atezolizumab for First-Line Treatment of Metastatic Nonsquamous NSCLC. N Engl J Med 2018;378:2288-301.

5. Cao R, Ma JT, Zhang SL, et al. Rational application of the first-line chemotherapy and immune checkpoint inhibitors in advanced nonsmall cell lung cancer: A meta-analysis. Cancer Med 2019;8:5033-46.

6. Hellmann MD, Paz-Ares L, Bernabe Caro R, et al. Nivolumab plus Ipilimumab in Advanced Non-Small-Cell Lung Cancer. N Engl J Med 2019;381:2020-31.

7. Lim SM, Hong MH, Kim HR. Immunotherapy for Nonsmall Cell Lung Cancer: Current Landscape and Future Perspectives. Immune Netw 2020;20:e10.

8. Reck M. Pembrolizumab as first-line therapy for metastatic non-small-cell lung cancer. Immunotherapy 2018;10:93-105.

9. Reinmuth N, Spigel D, De Marinis F, et al. Impower110: interim os analysis of a phase III study of atezolizumab (ATEZO) vs. platinum-based chemotherapy (chemo) as 11 treatment (tx) in pd-l1-selected NSCLC. Oncol Res Treat 2020;43:1-265.

10. Peters $S$, Ramalingam SS, Paz-Ares L, et al. Nivolumab (NIVO) + low-dose ipilimumab (IPI) vs. platinumdoublet chemotherapy (chemo) as first-line (1L) treatment (tx) for advanced non-small cell lung cancer (NSCLC): CheckMate 227 part 1 final analysis. Ann Oncol 2019;30:v851-v934.

11. Hellmann MD, Ciuleanu TE, Pluzanski A, et al. Nivolumab plus Ipilimumab in Lung Cancer with a High Tumor Mutational Burden. N Engl J Med
2018;378:2093-104.

12. Sezer A, Kilickap S, Gümüş M, et al. Cemiplimab monotherapy for first-line treatment of advanced nonsmall-cell lung cancer with PD-L1 of at least $50 \%$ : a multicentre, open-label, global, phase 3, randomised, controlled trial. Lancet 2021;397:592-604.

13. Paz-Ares L, Langer CJ, Novello S, et al. Pembrolizumab (pembro) plus platinum-based chemotherapy (chemo) for metastatic NSCLC: Tissue TMB (tTMB) and outcomes in KEYNOTE-021, 189, and 407. Ann Oncol 2019;30:v851-v934.

14. West H, McCleod M, Hussein M, et al. Atezolizumab in combination with carboplatin plus nab-paclitaxel chemotherapy compared with chemotherapy alone as firstline treatment for metastatic non-squamous non-smallcell lung cancer (IMpower130): a multicentre, randomised, open-label, phase 3 trial. Lancet Oncol 2019;20:924-37.

15. Jotte RM, Cappuzzo F, Vynnychenko I, et al. IMpower131: Primary PFS and safety analysis of a randomized phase III study of atezolizumab + carboplatin + paclitaxel or nab-paclitaxel vs. carboplatin + nab-paclitaxel as $1 \mathrm{~L}$ therapy in advanced squamous NSCLC. J Clin Oncol 2018;36:LBA9000.

16. Papadimitrakopoulou V, Cobo M, Bordoni R, et al. OA05.07 IMpower132: PFS and Safety Results with 1L Atezolizumab + Carboplatin/Cisplatin + Pemetrexed in Stage IV Non-Squamous NSCLC. J Thorac Oncol 2018;13:S332-3.

17. Paz-Ares L, Ciuleanu TE, Cobo M, et al. First-line nivolumab plus ipilimumab combined with two cycles of chemotherapy in patients with non-small-cell lung cancer (CheckMate 9LA): an international, randomised, openlabel, phase 3 trial. Lancet Oncol 2021;22:198-211.

18. Rizvi NA, Cho BC, Reinmuth N, et al. Durvalumab With or Without Tremelimumab vs Standard Chemotherapy in First-line Treatment of Metastatic Non-Small Cell Lung Cancer: The MYSTIC Phase 3 Randomized Clinical Trial. JAMA Oncol 2020;6:661-74.

19. Julian PT, Higgins JT, Jacqueline C, et al. Cochrane Handbook for Systematic Reviews of Interventions 2019; ISBN: 9781119536604.

20. Glenny AM, Altman DG, Song F, et al. Indirect comparisons of competing interventions. Health Technol Assess 2005;9:1-134, iii-iv.

21. Lumley T. Network meta-analysis for indirect treatment comparisons. Stat Med 2002;21:2313-24.

22. Borenstein M, Hedges LV, Higgins JP, et al. A basic introduction to fixed-effect and random-effects models for 
meta-analysis. Res Synth Methods 2010;1:97-111.

23. Moher D, Liberati A, Tetzlaff J, et al. PRISMA 2009 Checklist. Ann Intern Med 2009;151:264-9, W64.

24. Boyer M, Şendur MAN, Rodríguez-Abreu D, et al. Pembrolizumab Plus Ipilimumab or Placebo for Metastatic Non-Small-Cell Lung Cancer With PD-L1 Tumor Proportion Score $\geq 50 \%$ : Randomized, Double-
Blind Phase III KEYNOTE-598 Study. J Clin Oncol 2021; JCO2003579.

25. Borghaei H, Langer CJ, Paz-Ares L, et al. Pembrolizumab plus chemotherapy versus chemotherapy alone in patients with advanced non-small cell lung cancer without tumor PD-L1 expression: A pooled analysis of 3 randomized controlled trials. Cancer 2020;126:4867-77.

Cite this article as: Passiglia F, Galvano A, Gristina V, Barraco N, Castiglia M, Perez A, La Mantia M, Russo A, Bazan V. Is there any place for PD-1/CTLA-4 inhibitors combination in the first-line treatment of advanced NSCLC? - a triallevel meta-analysis in PD-L1 selected subgroups. Transl Lung Cancer Res 2021;10(7):3106-3119. doi: 10.21037/tlcr-21-52 
2. Cochrane Database search strategy

\section{Electronic search strategy}

We used a search strategy for randomized controlled trials (RCT) in Medline through Pubmed. The same search was then modified for RCT searches in other databases (Scopus, Controlled Cochrane Trial Register - CCTR).

The query was adapted from Robinson \& coll: [Robinson KA, Dickersin K. Development of a highly sensitive search strategy for the retrieval of reports of controlled trials using PubMed.

(" "Lung Neoplasms"[Mesh] OR "Carcinoma, Non-Small-Cell Lung"[Mesh] OR "lung cancer" "[tiab] OR "lung neoplasms"ttiab] OR "lung tumor"[tiab] OR "Non-small-cell lung cancer" [tiab] OR "Non small cell lung cancer"[tiab] OR NSCLC[tiab]) AND ("Neoplasm Metastasis"[Mesh] OR "Recurrence"[Mesh] OR "neoplasm metastasis" [ttiab] OR metasta* [tiab] OR "stage 4"[tiab] OR "stage IV"[tiab] OR "advanced" [tiab] OR Recurrence*"[tiab] OR relaps**tiab) AND ("Biomarkers

"Tumor/analysis" "Mesh] OR PD-L1, thab] OR PD LI[tiab]) AND (expression[tiab] OR “antibody" [tiab] "Pembrolizumab" (tiab] OR "aterlizus) AND ( pembrolizumab "Supplementary Concept] OR "nivolumab" [Supplementary Concept] OR "nivolumab" (tiab] OR "durzalumab" [Supplementary Concept] OR "durvalumab" [tiab] OR "ipilimumab" [Supplementary Concept] OR "ipilimumab"[tiab] OR "tremelimumab" [Supplementary Concept] OR "tremelimumab" "tiab] "Programmed Cell Death 1 Ligand 2 Protein" [Mesh] OR "PD-1" [tiab] OR “PD-L1"[tiab] OR "Drug Therapy/therapeutic wse"[Mesh] OR "Drug Therapy/therapy"[Mesh] OR "Drug Therapies"[tiab] OR "Chemotherapy" [tia

AND

("Randomized Controlled Trial "[Publication Type] OR "controlled clinical trial|"Publication Type] OR randomized[Title/Abstract] OR randomised[Title/Abstract] OR placebo[Title/Abstract] OR randomly[Tite/e/Abstract] OR groups[Title/Abstract] OR trial[Titile/Abstract] OR "drug therapy "[Subheading])]

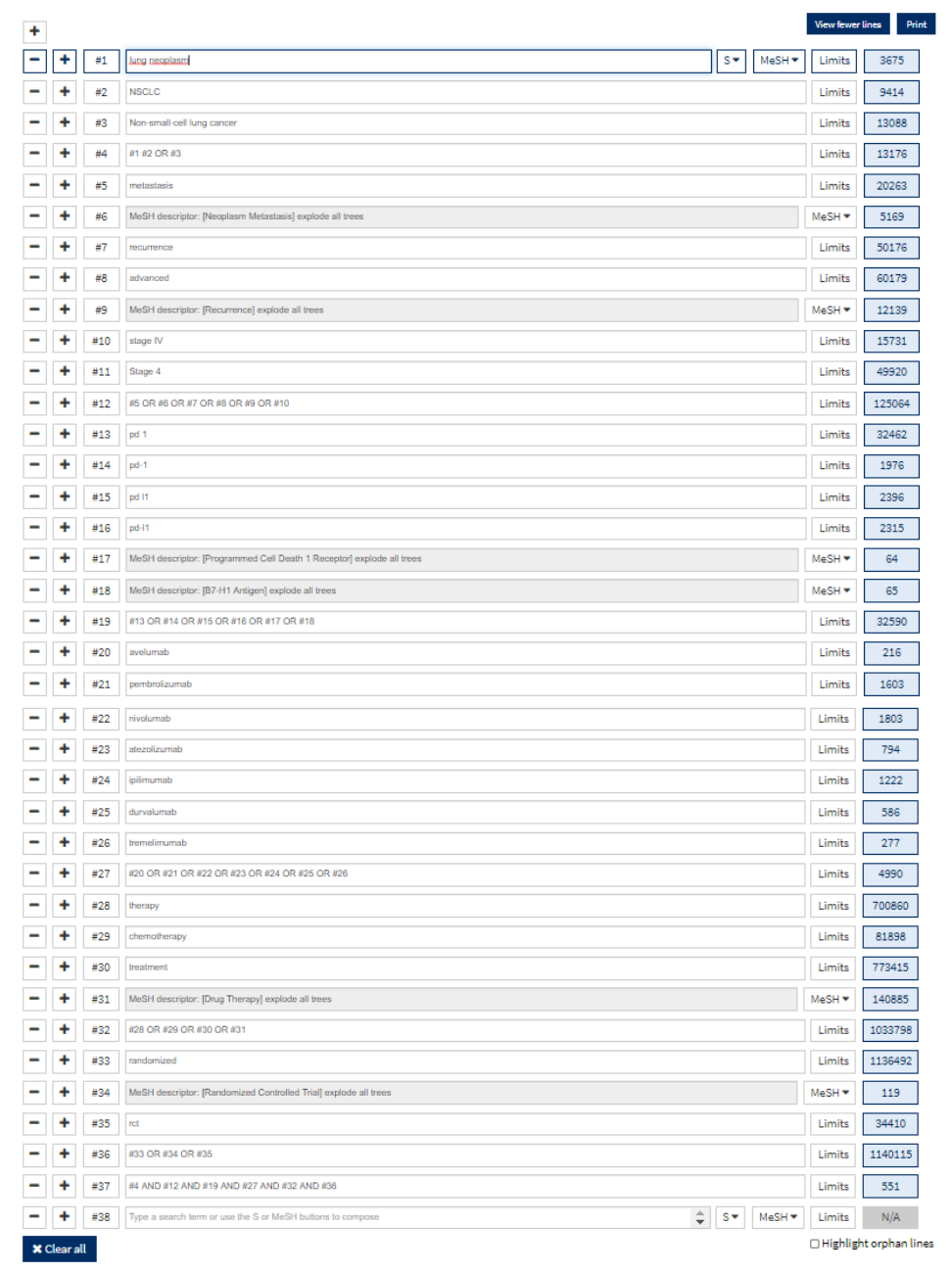

3. We used the free text strategy

mmunotherapy and advanced NSCLC survival response toxicity

(1)

Immunotherapy [tiab] AND NSCLC [tiab] AND meta-analysis [pt]

for RCT on-line searches in Clinical Trials registers (wwweclinicaltrials.goul

Figure S1 Electronic search strategy. 


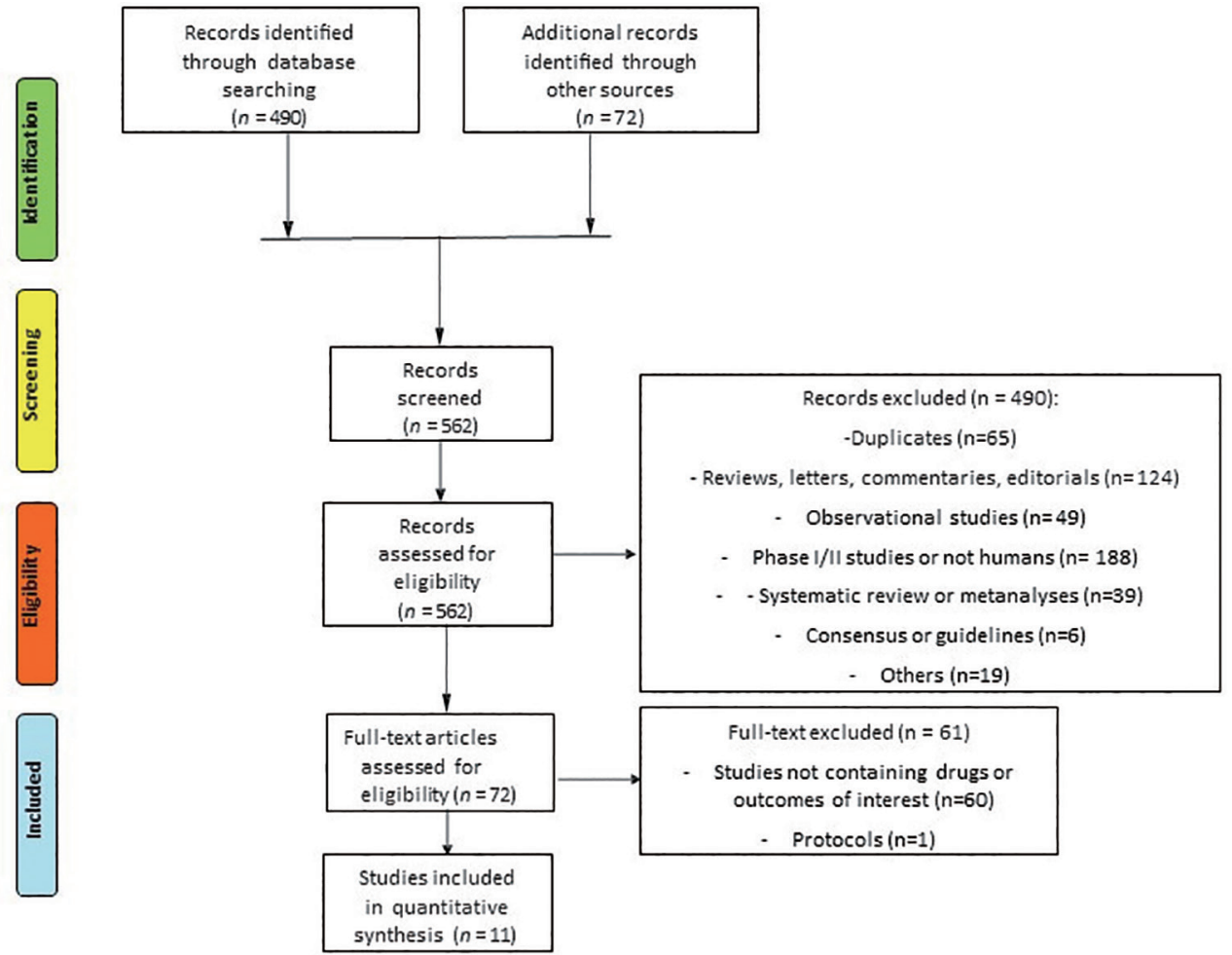

Figure S2 Flow diagram (CONSORT) of the included studies in the meta-analysis. 


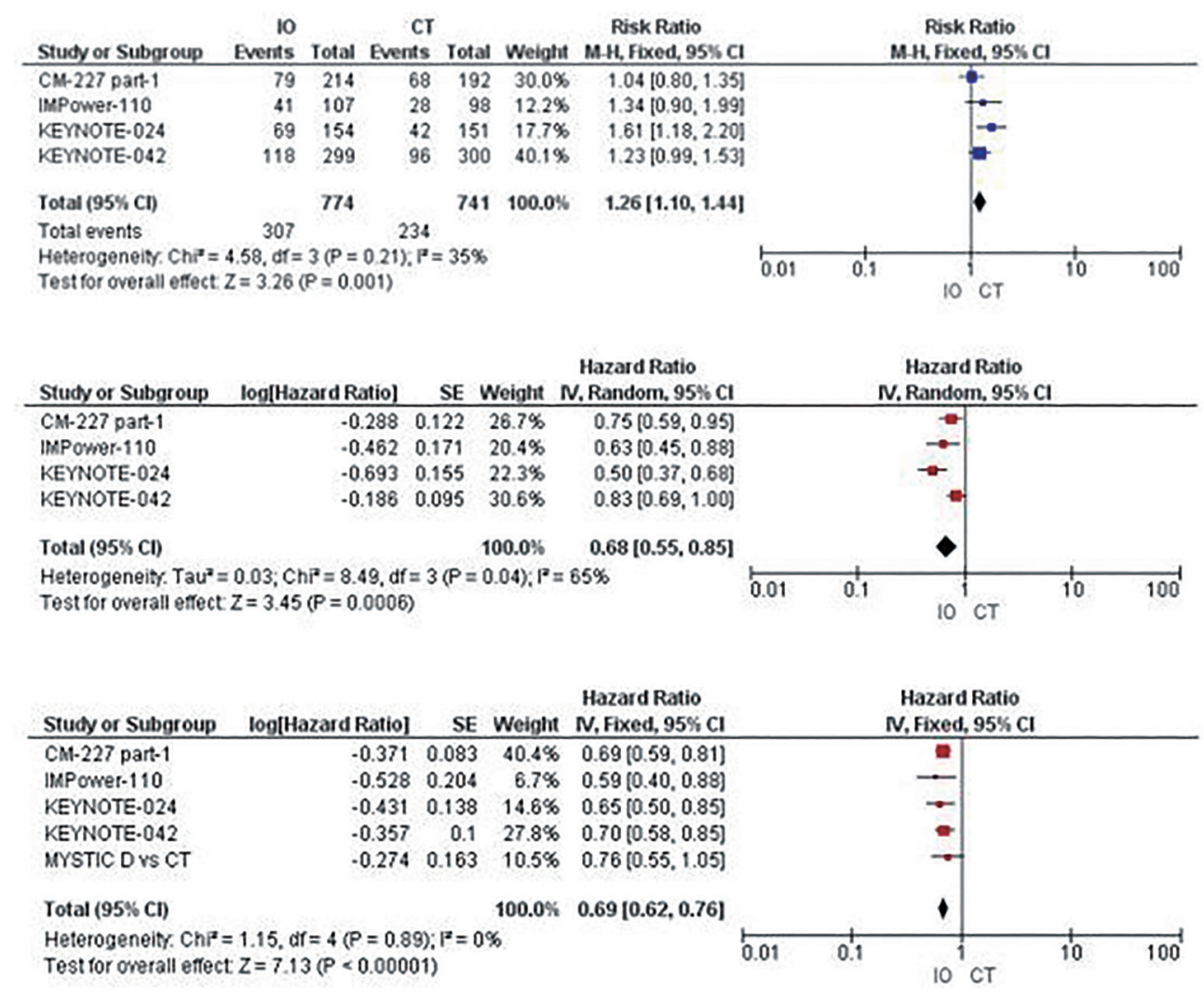

Figure S3 Forest plots of (A) RR of ORR, (B) HR of PFS and (C) OS of advanced NSCLC patients with tumor PD-L1 expression higher than 50\% undergoing IO versus CT alone. RR, risk ratio; ORR, objective response rate; HR, hazard ratio; PFS, progression-free survival; OS, overall survival; IO, single-agent immunotherapy; CT, standard platinum-based chemotherapy. 


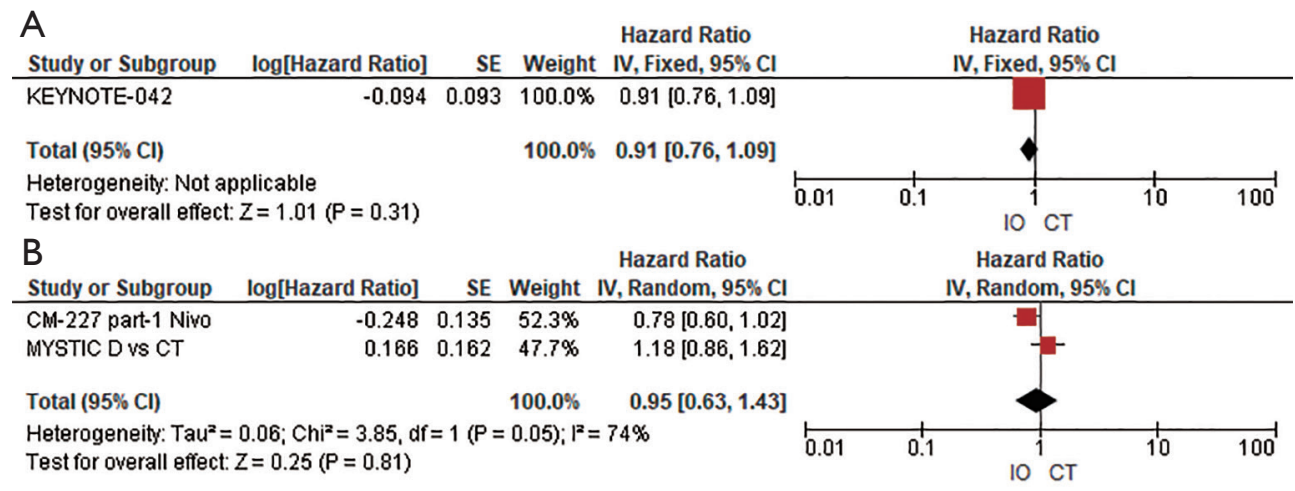

Figure S4 Forest plots of HR of OS of advanced NSCLC patients with tumor PD-L1 expression 1-49\% (A) and <1\% (B) undergoing IO versus CT alone. HR, hazard ratio; OS, overall survival; IO, single-agent immunotherapy; CT, standard platinum-based chemotherapy.

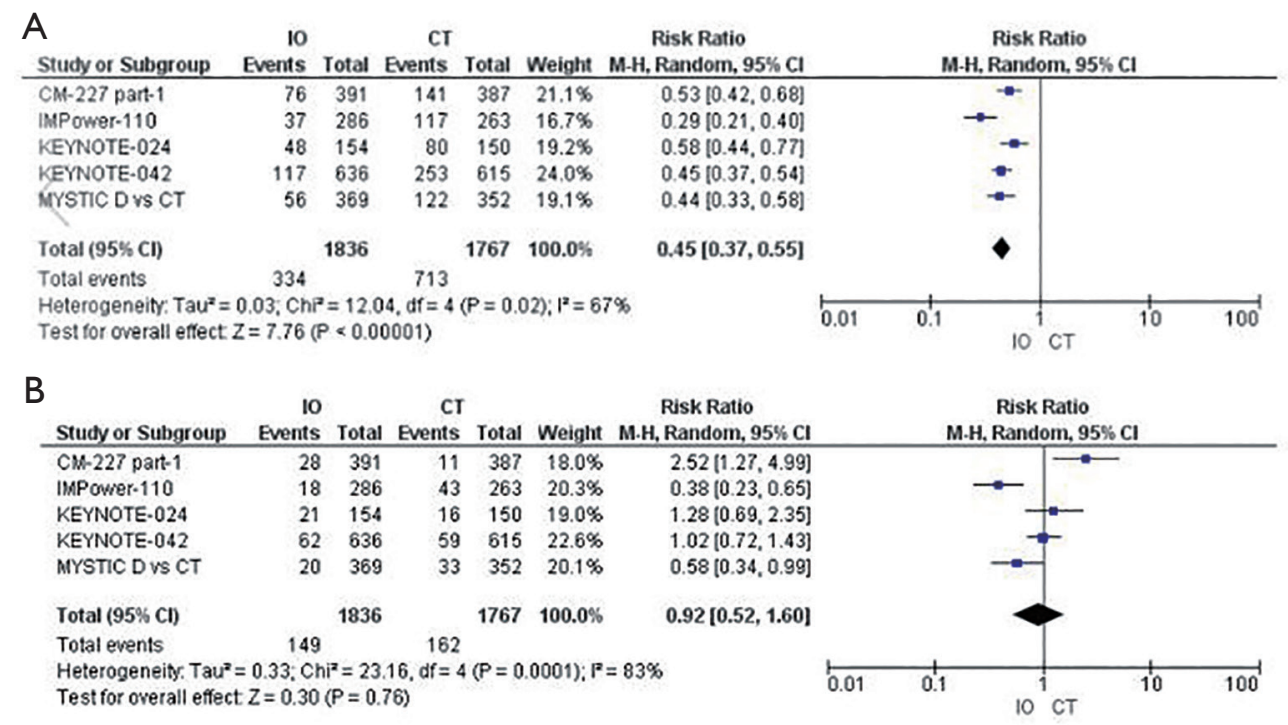

Figure S5 Forest plots of TRAEs (A) and discontinuation rate (B) in advanced NSCLC patients undergoing IO versus CT alone. TRAEs, treatment-related adverse events; IO, single-agent immunotherapy; CT, standard platinum-based chemotherapy. 


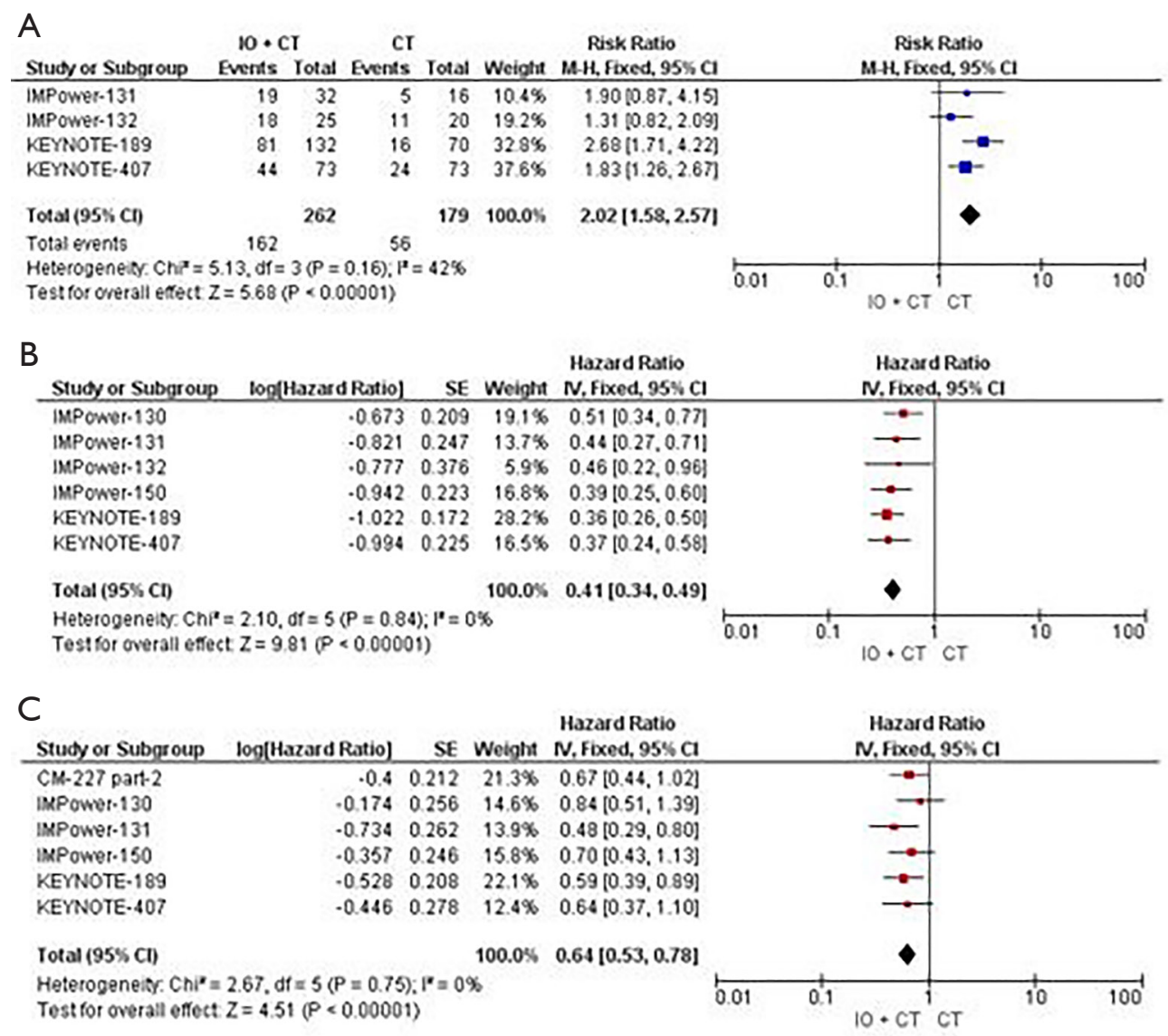

Figure S6 Forest plots of (A) RR of ORR, (B) HR of PFS and (C) OS of advanced NSCLC patients with tumor PD-L1 expression higher than 50\% undergoing IO + CT versus CT alone. RR, risk ratio; ORR, objective response rate; HR, hazard ratio; PFS, progression-free survival; OS, overall survival; IO, single-agent immunotherapy; CT, standard platinum-based chemotherapy. 


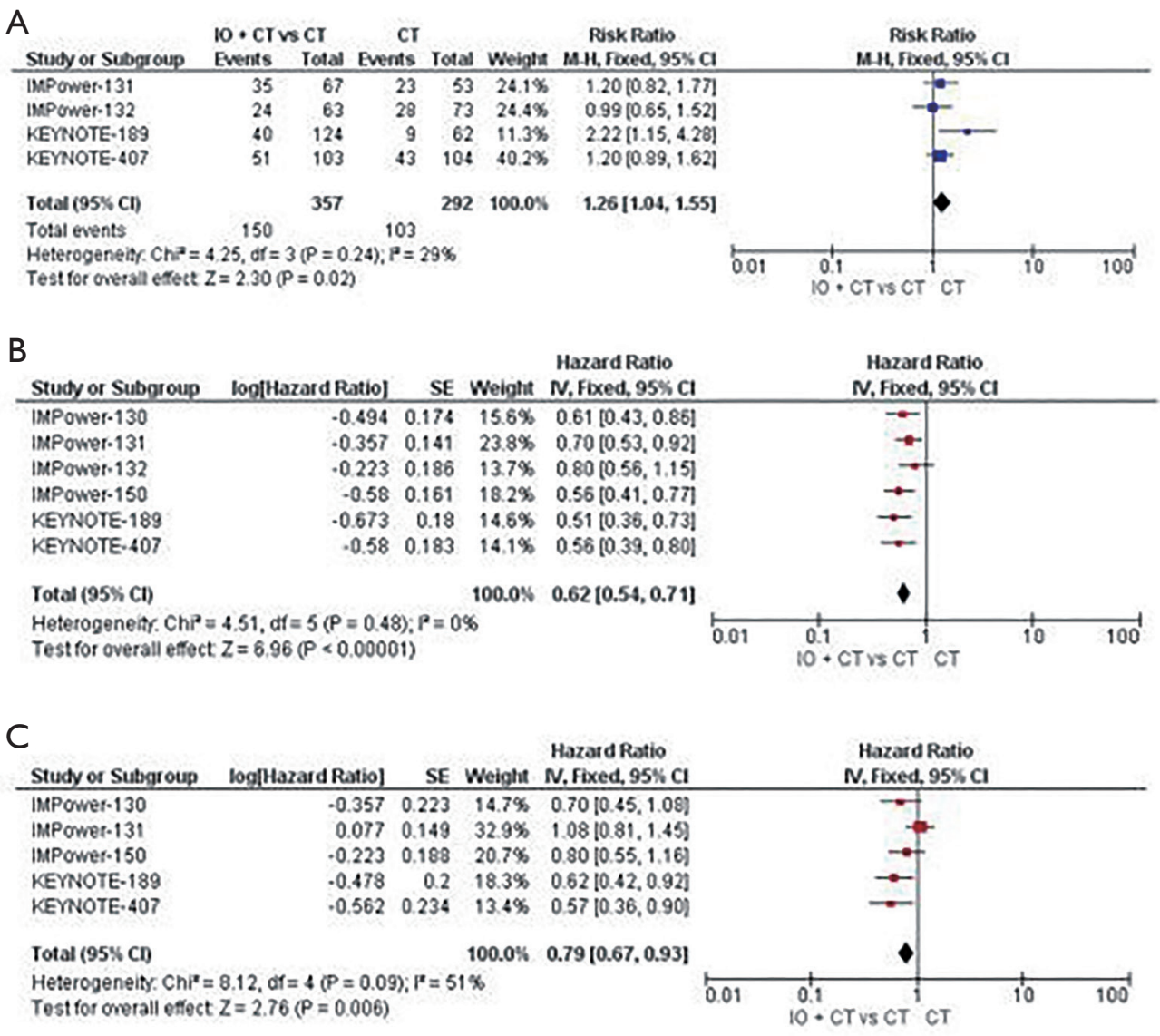

Figure S7 Forest plots of (A) RR of ORR, (B) HR of PFS and (C) OS of advanced NSCLC patients with tumor PD-L1 expression 1-49\% undergoing IO + CT versus CT alone. RR, risk ratio; ORR, objective response rate; HR, hazard ratio; PFS, progression-free survival; OS, overall survival; IO, single-agent immunotherapy; CT, standard platinum-based chemotherapy. 

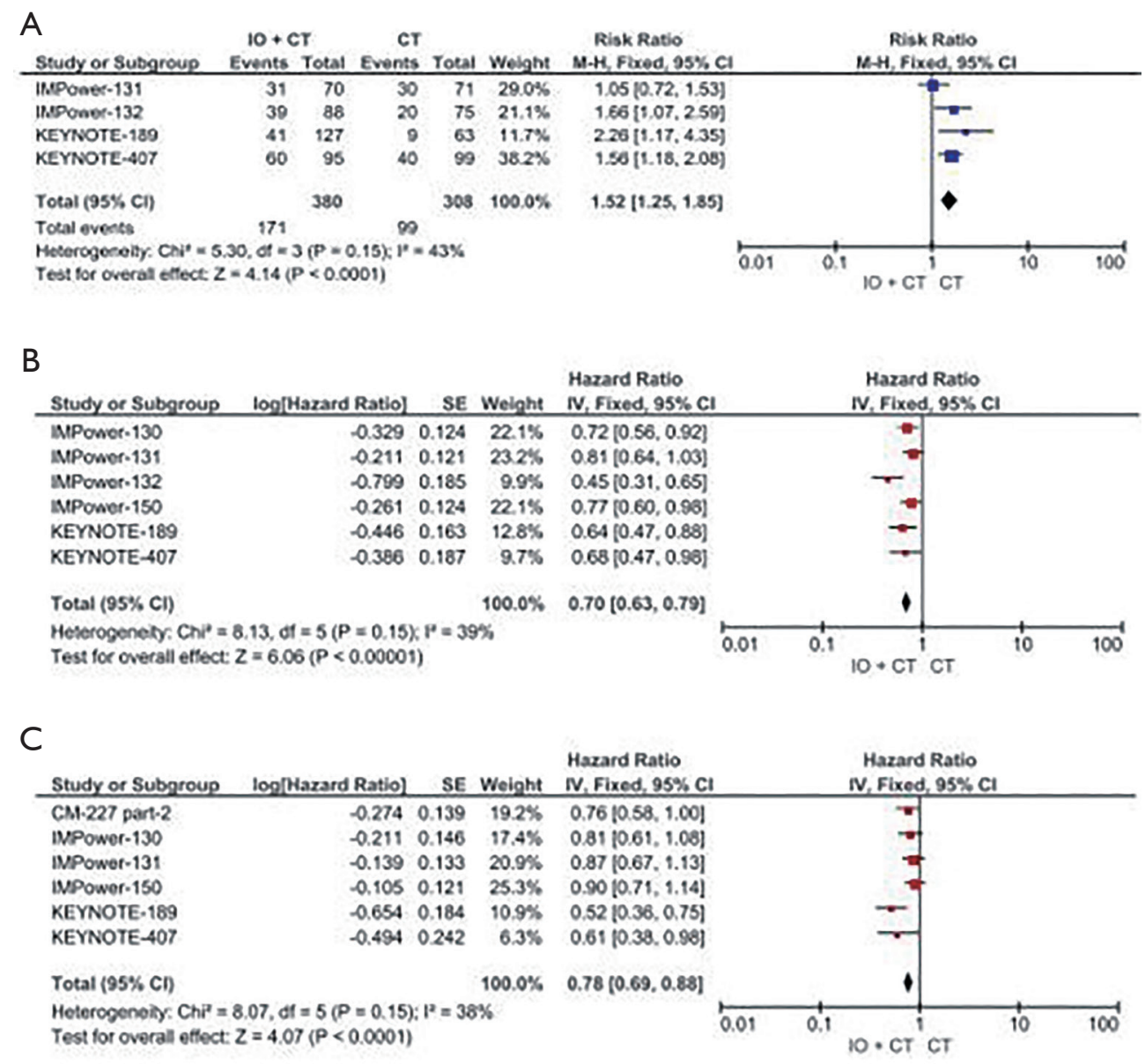

Figure S8 Forest plots of (A) RR of ORR, (B) HR of PFS and (C) OS of advanced NSCLC patients with tumor PD-L1 expression <1\% undergoing IO + CT versus CT alone. RR, risk ratio; ORR, objective response rate; HR, hazard ratio; PFS, progression-free survival; OS, overall survival; IO, single-agent immunotherapy; CT, standard platinum-based chemotherapy. 

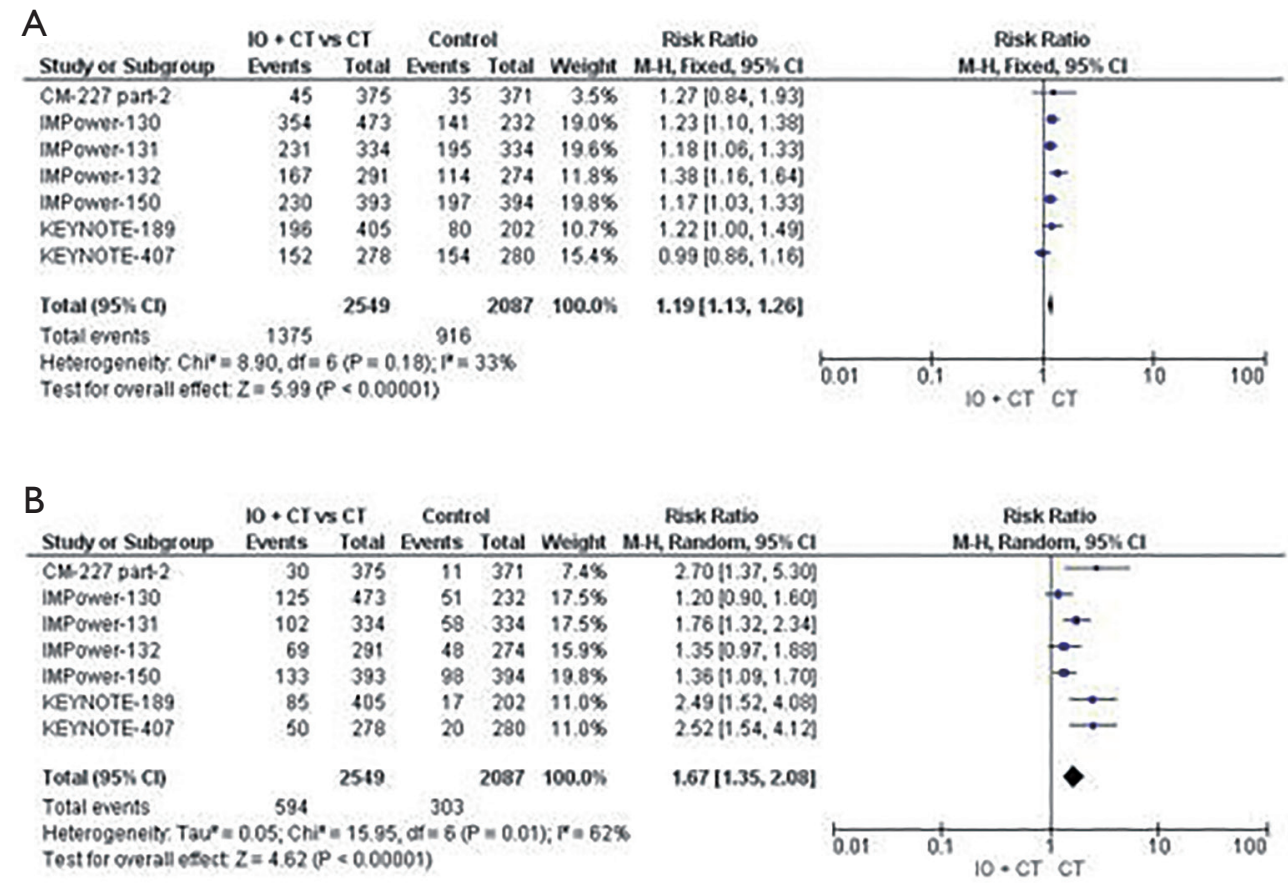

Figure S9 Forest plots of TRAEs (A) and discontinuation rate (B) in advanced NSCLC patients undergoing IO + CT versus CT alone. TRAEs, treatment-related adverse events; IO, single-agent immunotherapy; CT, standard platinum-based chemotherapy.

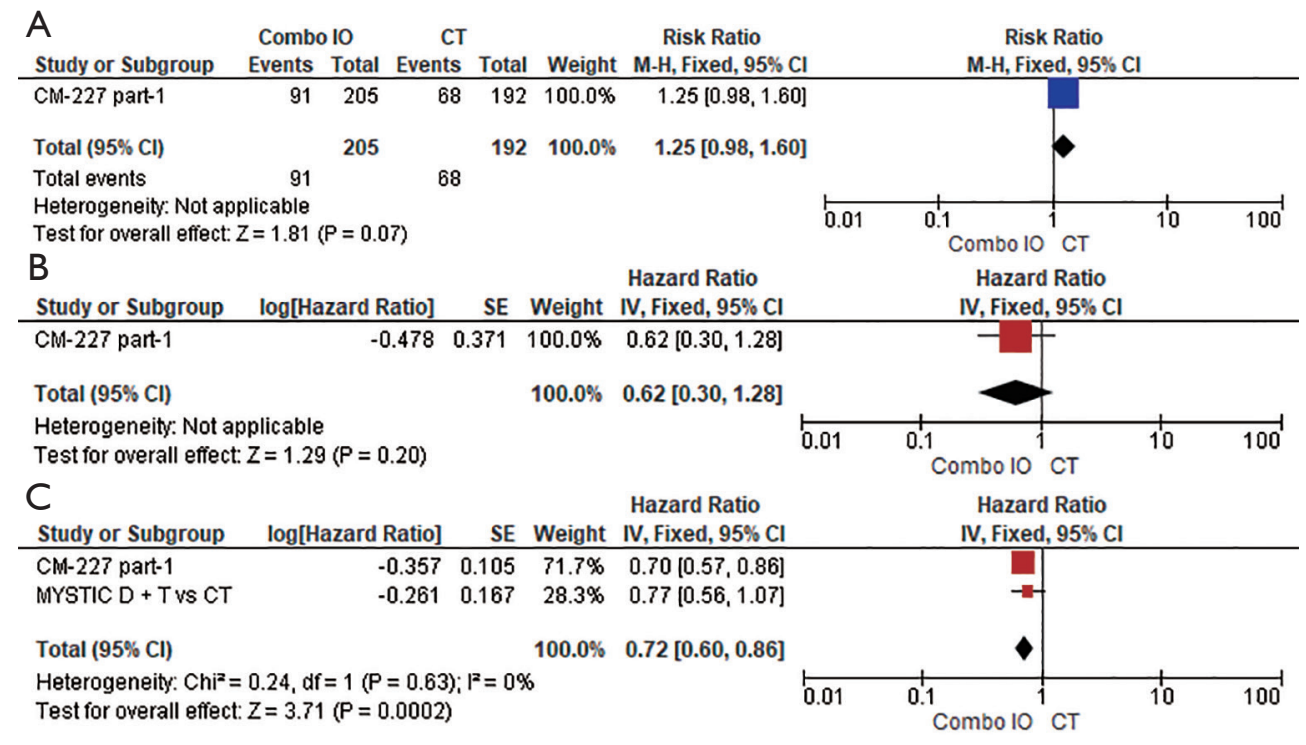

Figure S10 Forest plots of (A) RR of ORR, (B) HR of PFS and (C) OS of advanced NSCLC patients with tumor PD-L1 expression higher than 50\% undergoing Combo IO versus CT alone. RR, risk ratio; ORR, objective response rate; HR, hazard ratio; PFS, progression-free survival; OS, overall survival; Combo IO, immunotherapy combination regimens; CT, standard platinum-based chemotherapy. 


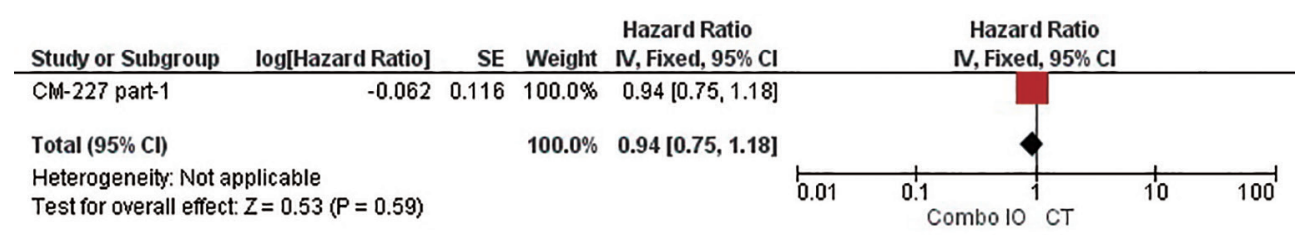

Figure S11 Forest plots of HR of OS of advanced NSCLC patients with tumor PD-L1 expression 1-49\% undergoing Combo IO versus CT alone. RR, risk ratio; ORR, objective response rate; HR, hazard ratio; PFS, progression-free survival; OS, overall survival; Combo IO, immunotherapy combination regimens; CT, standard platinum-based chemotherapy.

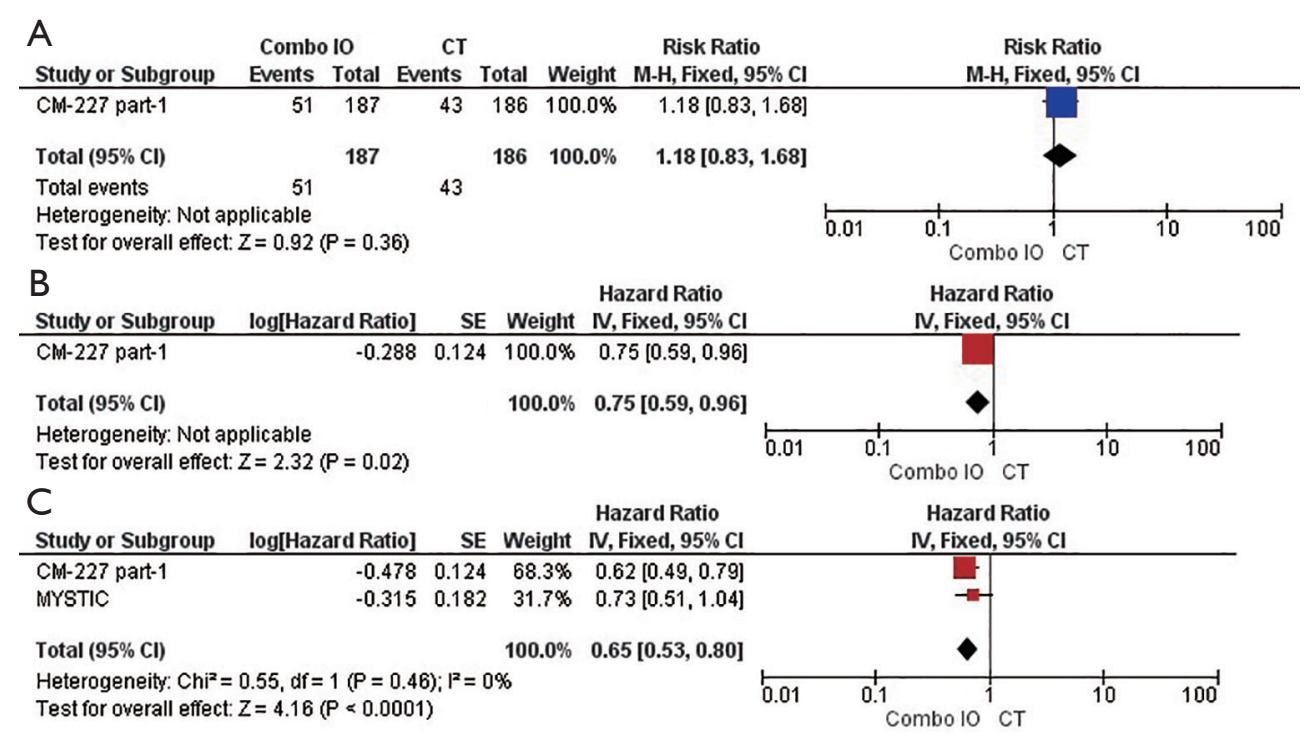

Figure S12 Forest plots of (A) RR of ORR, (B) HR of PFS and (C) OS of advanced NSCLC patients with tumor PD-L1 expression <1\% undergoing Combo IO versus CT alone. RR, risk ratio; ORR, objective response rate; HR, hazard ratio; PFS, progression-free survival; OS, overall survival; Combo IO, immunotherapy combination regimens; CT, standard platinum-based chemotherapy. 


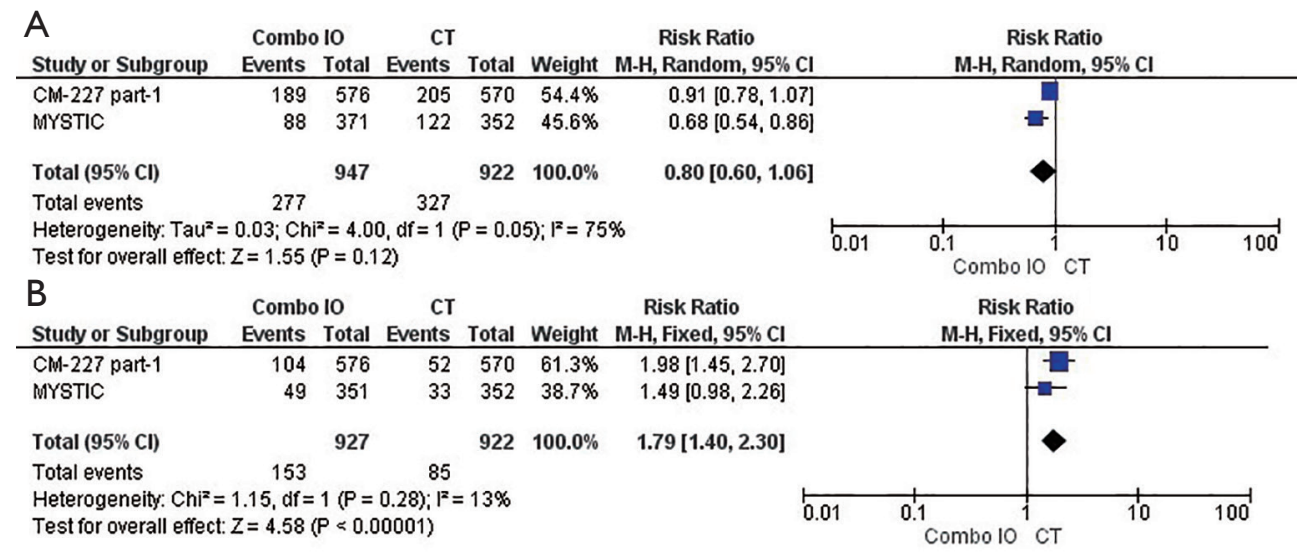

Figure S13 Forest plots of TRAEs (A) and discontinuation rate (B) in advanced NSCLC patients undergoing Combo IO versus CT alone. TRAEs, treatment-related adverse events; IO, single-agent immunotherapy; CT, standard platinum-based chemotherapy.

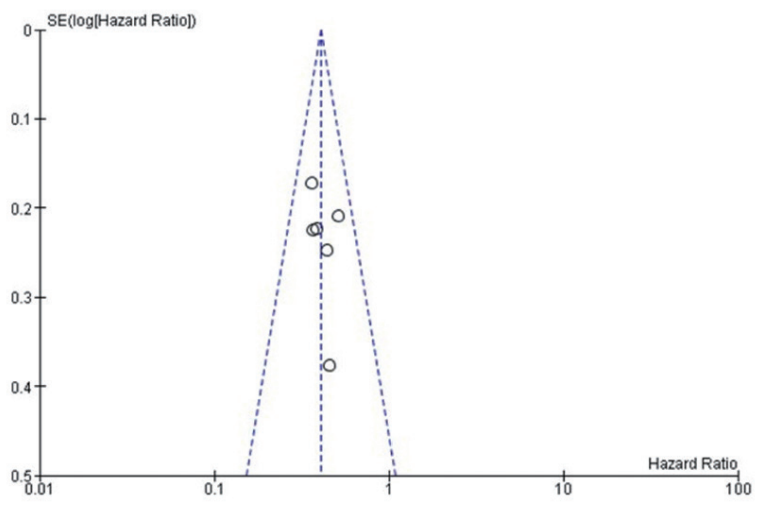

Figure S14 Funnel plot assessing the risk of publication bias across the studies. 


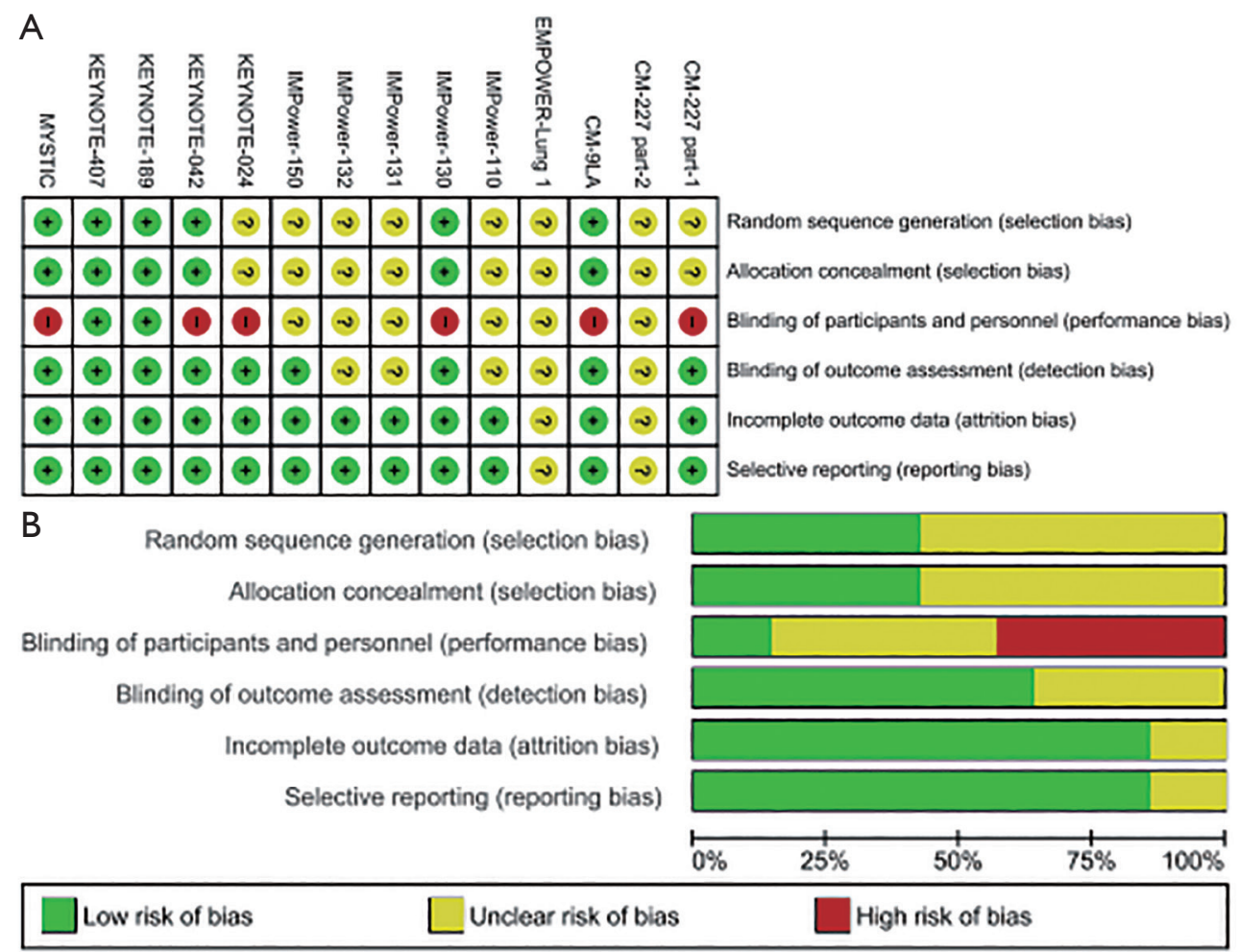

Figure S15 Risk of bias graph: review authors' judgements about each risk of bias item presented as percentages across all included studies (A); Risk of bias summary (B). 